This item was submitted to Loughborough's Research Repository by the author.

Items in Figshare are protected by copyright, with all rights reserved, unless otherwise indicated.

\title{
From graphene and topological insulators to Weyl semimetals
}

PLEASE CITE THE PUBLISHED VERSION

http://dx.doi.org/10.1142/9789814740371_0012

\section{PUBLISHER}

(C) World Scientific Publishing

\section{VERSION}

AM (Accepted Manuscript)

\section{PUBLISHER STATEMENT}

This work is made available according to the conditions of the Creative Commons Attribution-NonCommercialNoDerivatives 4.0 International (CC BY-NC-ND 4.0) licence. Full details of this licence are available at: https://creativecommons.org/licenses/by-nc-nd/4.0/

\section{LICENCE}

CC BY-NC-ND 4.0

\section{REPOSITORY RECORD}

Hills, Romilly D.Y., Matej Brada, Yang Liu, Michael P. Pierpoint, M.B. Sobnack, Wai M. Wu, and F.V. Kusmartsev. 2019. "From Graphene and Topological Insulators to Weyl Semimetals". figshare. https://hdl.handle.net/2134/26726. 
International Journal of Modern Physics B

(C) World Scientific Publishing Company

\title{
From graphene and topological insulators to Weyl semimetals
}

\author{
R. D. Y. Hills, M. Brada, Yang Liu, M. Pierpoint, M.B.Sobnack, W.M. Wu \\ Department of Physics, Loughborough University, Loughborough LE11 3TU, UK \\ F V Kusmartsev \\ Department of Physics, Loughborough University, Loughborough LE11 3TU, UK \\ E-mail: F.Kusmartsev@lboro.ac.uk \\ Received Day Month Year \\ Revised Day Month Year
}

\begin{abstract}
Here we present a short introduction into physics of Dirac materials. In particular we review main physical properties of various two-dimensional crystals such as graphene, silicene, germanene and others. We comment on the origin of their buckled two-dimensional shape, and address the issues created by Mermin-Wagner theorem prohibiting the existence of strictly two-dimensional, flat crystals. Then we describe main ideas which were leading to the discovery of two and three-dimensional topological insulators and Weyl fermions. We describe some of their outstanding electronic properties which have been originating due to the existence of the Dirac gapless spectrum. We also compare simplest devices made of Dirac materials. Analogies and differences between Dirac materials and optics are also discussed.

Keywords: Graphene, Silicene, Germanene, Phosphorene, Topological insulator, Weyl semimetal, Dirac cone, transistor, Landauer formalism
\end{abstract}

\section{Introduction}

The recent influx of study into the two-dimensional materials such as graphene, silicene, germanene and other has highlighted the field of gapless semiconductors ${ }^{1,2,3,4}$. After the discovery of graphene ${ }^{1,5}$ the physics community has exploded by novel ideas. Immediately, another new class of materials - two dimensional topological insulators(TI)- have been proposed ${ }^{6,7,8,9}$. After two dimensional TI was experimentally discovered in $2007^{10}$, it was recognised that there are their three dimensional analogues ${ }^{11}$ and Weyl fermions ${ }^{12,13,14,15,16,17}$. The understanding of the new physics of the topological materials was grounded on fundamental and seminal papers by Rashba who has first recognised the importance of the spin-orbital interaction in solids ${ }^{18,19}$. Indeed, the spin orbital interaction is responsible for the inversion of the bands in semiconductors. Therefore when we have a contact of two semiconductors having inverted band structure there semimetallic interface ${ }^{6}$ like that which exist in all topological materials on the surface ${ }^{10,11}$ arises. In addition, it was found that all these materials may be classified with the use of topological invariants, Chern 
numbers, which were first introduced in the electric transport in the Ref. ${ }^{20}$. The understanding of the topological properties of TI has lead to the discovery of the Quantum Spin Hall Effect(QSHE), which can arise naturally in the nano ribbon of novel two-dimensional materials. Their all, i.e. the already existing ribbon made of graphene, silicene, germanene and stanene are all two-dimensional topological insulators which have gap inside the ribbon and ballistic edge currents which may carry spin and charge degrees of freedom.

All these materials like graphene have Dirac spectrum, where the conduction and valence bands meet at a point known as a Dirac point ${ }^{5,6}$ and mass-less charge carriers follow a linear energy-momentum relation ${ }^{21}$. Due to the linear dispersion relation, high Fermi-velocity $\left(v_{f} \sim c / 300\right)$ and mass-less charge carriers, the quasiparticles in these materials can be modelled by the relativistic Dirac equation, see, for example the Ref. ${ }^{22}$ and materials may have different outstanding properties as like graphene, which has astounding electrical, thermal and optical characteristics. ${ }^{1,23,24,25}$. These properties have been used in various electronic (such as transistors ${ }^{26}$ and optoelectronic applications ${ }^{27}$.

\section{The shape of two-dimensional crystals: graphene, silicene, germanene, stanene, phosphorene and others}

The flat shape of graphene, silicene and other two-dimensional materials is a hexagonal honeycomb structure which is unstable due to a large surface energy. In other words; their surface to bulk ratio is very large. The displacement of atoms in the transverse direction decreases this ratio, or the surface energy. These displacements arise due to thermal fluctuations ${ }^{2,3,4}$, as first noted by Landau and Peierls. Usually graphene is found in the form of highly ordered pyrolytic graphite (HOPG), whereby individual graphene layers stack on top of one another to form a crystalline lattice. Carbon or silicene atoms are tightly packed into periodic array ${ }^{28}$ (cf. FIG. 1), and an $s p^{2}$ orbital hybridization - a combination of orbitals $p_{x}$ and $p_{y}$ that constitute the $\sigma$-bond. The final $p_{z}$ electron makes up the $\pi$-bond, and is key to the half-filled band which permits free-moving electrons ${ }^{29}$. In total, graphene has three $\sigma$-bonds and one $\pi$-bond. The right-hand portion of FIG. 1, emphasizes how small displacements of the sub-lattices A and B can be shifted in the z-direction ${ }^{30}$. This happens with all two-dimensional crystals such as graphene, silicene and germenane ${ }^{31,32}$. The existence of silicene was predicted in $2007^{33}$ and the material was first fabricated in $2012^{34}$. Indeed, the crystal has the hexagonal honycomb structure which is universally buckled as was first described in the paper ${ }^{31}$. A similarly structured 'two-dimensional', highly buckled material made from germanium is described in Ref. 30, 31, 32. This material 'germanene' was found in $2014{ }^{35}$. Recently, researchers have used Teflon to make flakes of phosphorene, which is the graphene-like material made of phosphorus ${ }^{36}$.

These materials have astonishing properties; due to their atomic bonding they are stronger than diamond, more conductive than copper, more flexible than rubber 

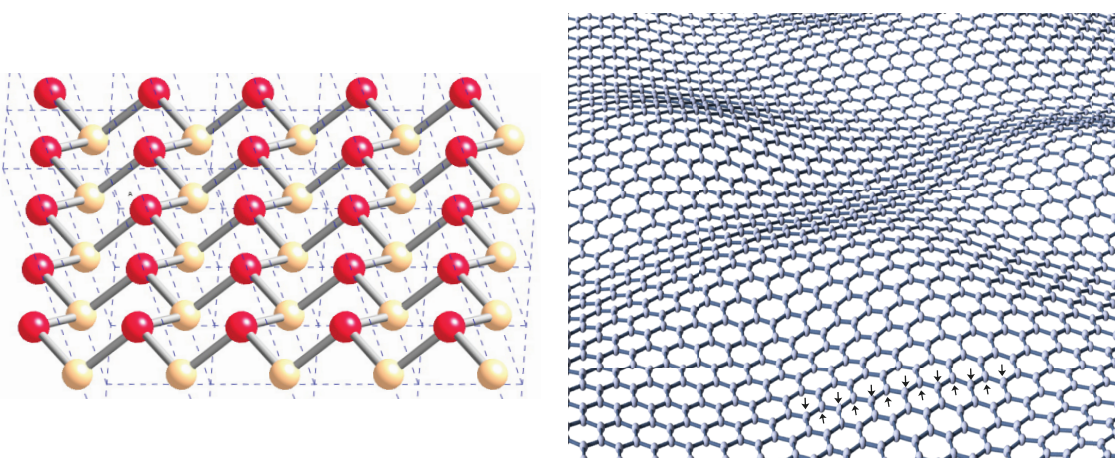

Fig. 1. The two-dimensional honeycomb lattice consisting of two A and B sublattices. The general shape of the two-dimensional crystals such as graphene, silicene, germanene and others are not flat due to Mermin-Wagner theorem ${ }^{30}$. Small quantum corrugations exist between the two sub-lattices, $\mathrm{A}$ and $\mathrm{B}$, which are shifted with respect to each other in a transverse direction by a distance of the order of some fraction of interatomic spacing. At high (room) temperature the displacements are dynamical and the shape looks nearly flat with corrugation remaining only on the large scale ${ }^{3}$. At low temperature microscopic quantum corrugations of $\mathrm{A}$ and $\mathrm{B}$ atoms indicated by small arrows are frozen, forming a glass state.

and optically transparent ${ }^{37,38}$. They exhibit anomalous quantum behaviour even at room temperature, such as Klein tunnelling, relativistic quantum Brownian motion 39,40,41,42 and the Quantum Hall Effect (QHE) ?. The 2010 Nobel Prize ${ }^{23}$ was the beginning of an adventure into the class of new Dirac materials - how to make these crystals (i.e. graphene, silicene germanene and other $2 \mathrm{D}$ crystals) useable is our main concern. Graphene sensors, transistors, and a number of electronic nano-devices are already emerging in laboratories every day ${ }^{43}$. Recently, the silicene transistor has been also developed and tested ${ }^{44}$.

The field-effect transistor (FET) was first proposed by John Bardeen and Willy Brattain at Bell Laboratories ${ }^{45}$. A transistor is the basic building block of many electronic circuits, normally acting as a logic gate or inverting amplifier in integrated circuits. According to Moore's law, the size of a circuit decreases twice every two years ${ }^{46}$. However, the speed of these devices has reached a bottleneck because the decreasing scale leads to an increase of the energy dissipation per unit area. The operating speed is also limited by the mobility and thermal conductivity of existing materials. The new 2D materials mentioned may resolve the issue.

In nature graphene layers stack together to form highly ordered pyrolytic 
graphite (HOPG) ${ }^{47}$. It has three $\sigma$ bonds and one $\pi$ bond. The $\sigma$ bonds are an $s p^{2}$ orbital hybridisation - a mixture of orbitals $\left(s, p_{x}, p_{y}\right)$ providing a strong binding force for its atomic neighbours. The $\pi$ bond corresponding to the $p_{z}$ orbital, forms a half-filled band that enables free-moving electrons, and hence exhibits a metallic characteristic ${ }^{48}$. Graphene is also capable of being combined with hydrogen or oxygen to form 'graphane', or other carbonic compounds respectively.

Wallace ${ }^{48}$ first studied the electronic band structure of monolayer graphite (graphene) with the simplest tight-binding model having a single hopping integral. He showed that its electronic spectrum is gapless at the Dirac point. A year later, Ruess and Vogt ${ }^{49,50}$ observed thin-film graphene oxide (GO) pieces using transmission electron microscopy (TEM). A single layer of graphene oxide was then discovered by Boehm et al. ${ }^{51,52}$ in the early 1960s, and it was Boehm et al. who first proposed the name 'graphene' 51,52 . Although research on graphene, graphene-like structures or epitaxial graphene films has continued for decades (cf. for example the Refs $53,54,55)$, it has significantly intensified since 2004, when Geim et. al. ${ }^{1}$ successfully segregated graphene flakes from graphite using the simple scotch-tape method ${ }^{56,56}$.

\section{Existing Fabrication Methodologies}

The different properties of graphene depend strongly upon its preparation. We focus on three methods in particular, include mechanical exfoliation from bulk graphite ${ }^{56}$, graphitisation of silicon carbide substrates (epitaxial growth) ${ }^{57,58}$, and chemical vapor deposition (CVD) on various transition metals ${ }^{59,60,61}$. The exfoliated graphene resides at the forefront in quality, followed by epitaxial graphene, and finally, various CVD graphene methods ${ }^{59,60,61}$.

The epitaxial graphene possess an intermediate range of values for the carriers mobility. Silicon carbide ( $\mathrm{SiC})$ is coated on a wafer, with high temperatures being required ${ }^{43}$. Recent studies of the Raman topography of epitaxial graphene, show that the electron mobility is highly dependent upon thickness and monolayer strain uniformity $62,63,64,65$. The highest mobility of epitaxial graphene reaches a value of $18,000 \mathrm{~cm}^{2} \mathrm{~V}^{-1} \mathrm{~s}^{-1}$ at room temperature and significantly larger at low temperatures. Also it was shown that the carrier mobility depends strongly upon the stacking of graphene layers ${ }^{62}$. The electron mobility is signficantly reduced in graphene/polymer hybrid materials ${ }^{66,67,68}$, although it is still remain high to improve significantly the materials for solar batteries. The suspended graphene can have a huge mobility, reaching values of up to $230,000 \mathrm{~cm}^{2} \mathrm{~V}^{-1} \mathrm{~s}^{-1} 69$. In the graphene industry ${ }^{43}$ chemical vapor deposition (CVD) is usually used. The charge carrier mobility of CVD graphene is typically orders of magnitude lower than for exfoliated graphene ${ }^{70,71,68,69}$ or theoretical estimations for Dirac materials ${ }^{72,73}$. The CVD graphene mobility can reach $2,700 \mathrm{~cm}^{2} \mathrm{~V}^{-1} \mathrm{~s}^{-1}$. With the grain boundary ${ }^{74}$ it will be roughly three or four times smaller ${ }^{74,62}$. Mobility is a measure of how fast the carriers propagate in an electric field. Its value depends upon the type of 
material and the interaction with substrates. After the process of annealing, the mobility of epitaxial graphene at room temperature $(\approx 300 \mathrm{~K})$ can reach values upwards of $100,000 \mathrm{~cm}^{2} \mathrm{~V}^{-1} \mathrm{~s}^{-1} 26,27$. This can be even higher in a perfect structure according to some theories ${ }^{46}$, or even tend to infinity when chemical potential is at the Dirac point ${ }^{73}$. However, it was found that even for the CVD graphene ${ }^{74}$ at this specific Dirac point, there should be a universal 'minimum' quantum conductivity 75 :

$$
\sigma_{0}=\frac{4 e^{2}}{\pi h}
$$

Here the standard conductance quantum, $G_{0}=2 e^{2} / h^{76}$. The exfoliated graphene at the Dirac point has the double conductance quantum or half of the quantum resistance $R_{0}$ equal to $R_{0}=1 / G_{0}=12.9 k \Omega^{77}$.

At the Dirac point, when the concentration of electrical carriers tends to zero even at room temperature, the transport may be ballistic, i.e. without much scattering at all ${ }^{6,71}$. Also the heat in suspended graphene is conducted by ballistic phonons. ${ }^{78}$. The thermal conductance of graphene on a substrate is significant downgrade, since the number of scattering channels increases. The thermal conductance also decreases with an increased number of graphene layers. Analysing nano-thermal images, it was found that the mean-free-path of thermal phonons in graphene on a substrate, is smaller than $100 \mathrm{~nm}$. According to Prasher ${ }^{79}$, the thermal conductivity of graphene on a silicon-dioxide substrate can reach around 600 $\mathrm{Wm}^{-1} \mathrm{~K}^{-1}$, incidentally higher than copper. Other papers provide even higher values for the in-plane thermal conductivity, but of the same order of $1,000 \mathrm{Wm}^{-1} \mathrm{~K}^{-1}$ 80,81 . Due to this high in-plane thermal conductivity, the heat dissipation from graphene is also affected by the heat flow across its interfaces. Thus, in-plane heat transport for graphene is associated with acoustic phonons ${ }^{78,81}$. The mechanism of heat transport across graphene interfaces in the cross-plane direction will depend on the structure and quality of substrate et al. ${ }^{82}$. Young's modulus for graphene is about $1 \mathrm{TPa}{ }^{26,27}$ - it is almost the strongest material in the world ${ }^{47}$.

It is of interest that in epitaxial graphene grown on $4 \mathrm{H}-\mathrm{SiC}$ face-terminated carbon; various nano-structures resembling charged 'bubbles' and 'domes' have been discovered ${ }^{65}$. These graphene nano-structures, domes and bubbles are having a few tens of nanometres (150-200 $\mathrm{nm}$ ) in size. The free-standing nature of the graphene layer within the domes and bubbles, with heights on the order of 5-12 nm may be useful for many applications. Inside the bubbles and substrate there arises complex charge redistribution; in fact, the graphene bubble-substrate interface forms a charged capacitance. The strength of the electric field inside the bubble-substrate interface may lead to a minigap of the order of $5 \mathrm{meV}$. The similar charge redistribution may arise Graphene-polymer(P3HT) hybrids useful for photovoltaic applications $^{66,67}$. 


\section{The physical properties of graphene}

The absorption spectrum of a single graphene layer is quite flat for light, with a wavelength $\lambda$ ranging from $300 \mathrm{~nm}$ to $2,500 \mathrm{~nm}$, and a broad peak intensity in the ultraviolet region. The peak arises due to the van Hove singularity in the energy density of states, and its transmittance can be expressed in terms of the fine-structure constant ${ }^{83}$. It absorbs a mere $2.3 \%$ of visible light, thus possessing a high transparency ${ }^{38}$, i.e. it is a suitable material for photonic devices. Being transparent to visible light due to its atomic thickness, such a material can be made available for small screen devices $38,47,79$. There is also common belief that graphene's true potential lies in photonics and opto-electronics, as discussed by Nair et al. ${ }^{83}$ and Bonaccorso et al. ${ }^{84}$. Stoehr et al. ${ }^{85}$ observed white optical luminescence of graphene, when subject to picosecond infrared laser pulses - this creates a high density electron-hole plasma. The luminescence arises due to electron-hole recombination, created in a broad energy range. There are two relaxation mechanisms observed in the graphene electron-hole plasma ${ }^{86}$. A faster one is usually associated with carrier to carrier intra-band collisions, and a time scale of about of 100 femtoseconds. The slower one is via phonon emission, which has only a picosecond timescale. This corresponds to electron inter-band relaxation ${ }^{87,88}$. This is why graphene may potentially be an ideal photonic and opto-electronic material ${ }^{31,30,32}$.

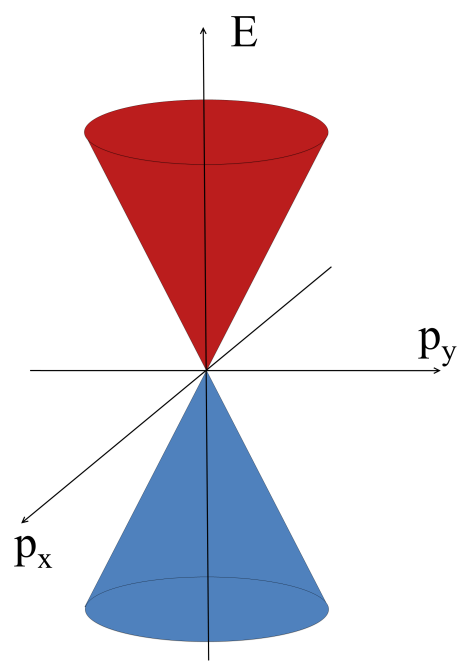

Fig. 2. A linear energy-momentum spectrum near the Dirac point is exhibited for graphene, $E= \pm v_{F} \sqrt{p_{x}^{2}+p_{y}^{2}}$, where $v_{F}$ is the Fermi velocity and $\left(p_{x}, p_{y}\right)$ is the momentum of an electron. The Fermi energy level $E_{F}$ coincides with the Dirac point - where the density of states is equal to zero. Electrons occupy all states in the lower cone, with the upper cone remaining unoccupied.

The graphene honeycomb lattice consists of two carbon sub-lattices (A and B) 
per unit cell ${ }^{47}$ (cf. Figure 1). There are six Dirac points in the Brillouin zone, with a linear energy-momentum relationship. ${ }^{48}$,

$$
E= \pm v_{F} \sqrt{p_{x}^{2}+p_{y}^{2}},
$$

where $v_{F}$ is Fermi velocity, and $p_{x}$ and $p_{y}$ are the momenta in ' $\mathrm{x}$ ' and ' $\mathrm{y}$ ' directions respectively. A single Dirac cone for the graphene energy spectrum is shown in Figure 2, which is a direct contact of the conduction and valence bands, a zero band-gap situated at the Dirac points ${ }^{43,48}$ and zero effective mass.

They belong to two valleys, which may be well described by the relativistic Dirac Hamiltonian

$$
\hat{H}=v_{F}\left(\sigma_{x} \tau_{z} \hat{p}_{x}+\sigma_{y} \hat{p}_{y}\right),
$$

where $\left(\sigma_{x}, \sigma_{y}\right)$ are $2 \times 2$ Pauli matrices associated with A and B sublattices, $\hat{p}_{x}$ and $\hat{p}_{y}$ refers to the momentum operators. The eigenvalues of this $4 \times 4$ matrix Hamiltonian, $H$, immediately give the Dirac spectrum in Eq.(9). In general, quantum behavior is normally exhibited at low temperatures; such processes include the QHE, where the transverse Hall resistance is quantised. However, with graphene, the possibility has now arisen to study such quantum behavior even at room temperature 39,40 . Some of the most recent experimental research ${ }^{39}$ has reported observation of the QHE within a graphene monolayer, displaying the quantum resistance at room temperature. The fractional QHE in graphene is associated with the electronelectron interaction, and a four-fold zero energy degenerate state is originated due to the existence of two degenerate sub-lattices (A and B). Both fractional and anomalous QHE have been observed within the single monolayer, as well as bilayer graphene ${ }^{39,40}$.

Graphene provides a new quantum feature with regards to perfect transmission through barrier $90,91,92,93$. In classical mechanics, an electron with kinetic energy $K$ cannot penetrate a potential barrier $U$ when $K<U$. Interestingly, quantum theory shows that there is a certain probability of transmission, even when $K<U$. Graphene electrons can tunnel through the barrier with perfect transmission ${ }^{91}$ a process called Klein tunnelling ${ }^{90}$. Katsnelson et al. further explains that the barrier repels electrons from the left hand side, with a hole inside the barrier ${ }^{90}$. As an electron moves through the barrier, it first transforms into a hole on the left hand side. The hole is then transformed again into an electron on the right hand side of the barrier. As a result, this generates a continuous transmission probability spectrum across the entire barrier ${ }^{90}$. Overall, it looks as if electrons penetrate the square potential wall, without restriction being placed upon on its height. Recently, Zalipaev et al. discovered that different shapes of potential wells (e.g., parabolic or smooth, non-rectangular) would influence the tunnelling behaviour of an electron in graphene (cf. for details ${ }^{42}$ ). There is a drastic difference between tunnelling through smooth and rectangular barriers. The Klein tunnelling features are clearly displayed only for a rectangular barrier. The type of the tunnelling behavior depends on the energy of the particle relative to the barrier height and the angle at which the 


\section{Klein Tunneling}

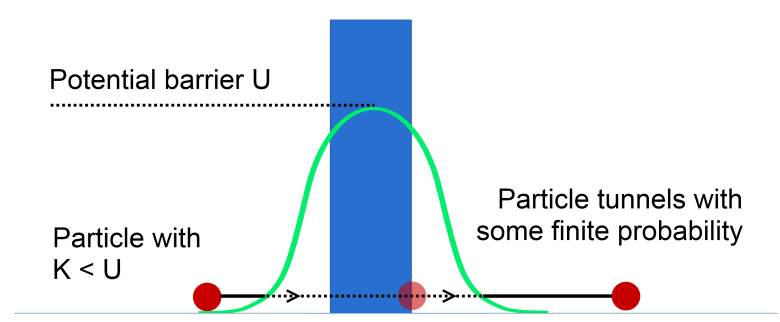

Fig. 3. Klein tunnelling with perfect transmission through the barrier is schematically shown. In classical mechanics, a particle with kinetic energy $K$ is incapable of surpassing a potential barrier $U$, for the condition $K<U$. However, quantum mechanics allows a particle to tunnel through the barrier with some finite probability, even when $K<U$. This becomes most unusual in the case of graphene. For certain angles, a particle can tunnel through the barrier with perfect transmission.

particle approaches the potential. When the energy of the particle is close to the top of the barrier one observes conventional tunnelling. For intermediate energy the smooth potential acts as Fabry-Perot interferometer. While for energies close to the Dirac point there arise confined bound states ${ }^{42}$. The confinement effect arising at small energies for the smooth potential of the special waveguide geometry have been also found in Refs ${ }^{94,95,96}$. The conducting waveguide channel in electronic devices is usually created by a gate voltage applied to graphene. That leads to the lateral confinement of electrons in the channel. However, electrostatic potentials of the circular form, which are very smooth, i.e. do not have exponentially decaying tails may possess a threshold for the appearance of the zero-energy confined state 97,98. This may lead to unusual Aharonov-Bohm (AB) effect in graphene, e.g. the 
fractional AB effect arising in strongly correlated electrons $99,100,101,102$.

Quantum capacitance is one of the most interesting properties of a graphene capacitor, as reported by $\mathrm{Yu}$ et al. ${ }^{103}$. Total capactiance $C$ in graphene can be found by $1 / C=1 / C_{e s}+1 / C_{q}$, where $C_{e s}$ is related to classical electrostatics, and $C_{q}$ is the quantum capacitance. $C_{q}$ is proportional to the chemical potential $\mu$, and also the number electron states (spin up/down) near the $\mathrm{K}$ and $\mathrm{K}$ ' points of the brillouin zone (BZ) ( $\mathrm{Yu}$ et al. ${ }^{103}$ ). The DoS in two dimensional graphene is given by ${ }^{40}$ :

$$
D(E)=\frac{2|\mu|}{\pi \hbar^{2} v_{F}^{2}}
$$

Then the quantum capacitance is given by:

$$
C_{q}=A e^{2} D(E)=\frac{2 A e^{2}|\mu|}{\pi \hbar^{2} v_{F}^{2}}
$$

where $A$ is the surface area of the capacitor electrodes ${ }^{103}$. For zero applied electric field, the chemical potential $\mu$ and density of states (DoS) are very small. At low temperatures the dependence of the chemical potential (the Fermi energy, $\epsilon_{F}$ ) on the electron density $n$ has very simple form:

$$
\mu=\epsilon_{F}=\frac{h v_{F}}{2} \sqrt{\frac{n}{\pi}}
$$

For epitaxial graphene, which is usually intrinsically doped to the electron density $n=10^{12} \mathrm{~cm}^{-2}$, we can immediately find both the Fermi energy and the quantum capacitance. Taking the Planck constant equal to $h=6.5810^{-16} \mathrm{eV}$ and the Fermi velocity equal to $v_{F}=10^{6} \mathrm{~m} / \mathrm{s}$ we obtain that the Fermi energy there will be equal to $\epsilon_{F}=18 \mathrm{meV}$. The Fermi energy is shifted by this value above the Dirac point. The quantum capacitance will be equal to $C_{q}=18 \mathrm{mF} / \mathrm{cm}^{2}$. Therefore, $C_{q}$ is small also, when the density is small and it is the dominant contribution to the total capacitance ${ }^{73}$. This is the so called graphene quantum capacitance effect (cf. Xia et al. ${ }^{104}$ and Giannazzo et al. ${ }^{105}$ ). However, as an applied electric field increases, more electrons occupy the conduction band, and $\mu$ shifts to an upper value. $C_{q}$ becomes larger compared to $C_{e s}$, with the quantum capacitance effect becoming less important. Recent studies have discovered that $C_{q}$ can be reduced significantly due to disorder of the graphene structure, as mentioned by Li et al. ${ }^{106,107}$.

\section{Applications of Graphene Devices}

Some common applications of graphene to electronic devices are discussed in this section. These include various graphene sensors ${ }^{108,109,110,111,112}$, photonic optics 43,113 and capacitors. 


\subsection{Graphene Sensor}

Impermeability is one of the most outstanding properties of graphene, since the carbon atoms are closely packed with one another ${ }^{47}$. The conductivity of graphene is sensitive to impurities, and can change easily when other molecules or substrates become attached to it. The variation of such conductivity due to molecular attachment is the principle foundation for molecular sensors ${ }^{110}$. Graphene, with its two-dimensional surface, provides a platform upon which tiny particles can attach. The deviation of electrical resistivity, caused by the attached molecules (impurities), can be measured by the Hall Effect ${ }^{109,110}$. In Singapore, He et al. ${ }^{109,110}$ have successfully developed bio-sensors and gas sensors using such a method. In the near future, graphene is also likely to be a plausible candidate for the magnetoresistance sensors ${ }^{112}$

\subsection{Graphene Photonic Devices}

Recently, the dielectric sandwich - two layers of graphene with dielectric filling has been used as an optical signaling modulator (see, the Fig.4) ${ }^{114,115}$. Gosciniak et al. ${ }^{114}$ estimate that this graphene optical modulator can reach speeds of up to $850 \mathrm{GHz}$, with $3 \mathrm{~dB}$ modulation and small losses. Liu et al. ${ }^{115}$ have also reported a wide absorption range of $1.35-1.60 \mu \mathrm{m}$ in wavelength.

A waveguide is a physical channel which traps light, guiding it through a designated path ${ }^{116}$. For example, graphene nano ribbon are similar to fiber-optic cable, which is a common waveguide for the communication of light signals - its high refractive index $n_{o p}$ trapping light inside the fiber ${ }^{117}$. Zhang et al. ${ }^{116}$ have studied the wave-modes of the graphene quantum well, identifying energy dispersion relations associated with Klein tunneling and classical wave-modes ${ }^{116}$. Zhang et al. 116 further note an absence of the third order classical, and first order tunneling wave-modes.

Graphene plasmonic waveguides have become an essential component for integration with logical devices ${ }^{118,119}$. Kim et al. ${ }^{118}$ have studed the plasmonic waveguide for a dielectric substrate coated on graphene, discovering little optical loss and very fast operating speeds. They show that at the peak wavelength $\lambda=1.31 \mu \mathrm{m}$, the transmission ratio is around $19 \mathrm{~dB}$ for the TM mode ${ }^{118}$.

As we have already highlighted upon, saturable absorption refers to an absorption of photons decreasing as the light intensity increases ${ }^{120,121}$ It is usually applied via the mode-locked laser ${ }^{122}$. Many semiconductors exhibit saturable absorption, but are not as sensitive as graphene ${ }^{120}$. The basic idea is as follows - a number of excited electrons occupy the conduction band during high intensity exposure, and electrons in the valence band are no longer able to absorb photons due to the Pauli exclusion principle ${ }^{122}$. This property originates from the nonlinear susceptibility of graphene for a short response time ${ }^{123}$. In application, a saturable absorber can 
be used to transform a continuous wave to a very short wave pulse ${ }^{123}$. Generally speaking, monolayer graphene provides a high saturable absorption coeffcient, and recently, some research has uncovered that CNTs may also be suitable candidate for a saturable absorber ${ }^{121,124}$. Bao et al. ${ }^{122}$ also report that a single layer graphene (SLG) saturable absorber can provide around $66 \%$ modulation depth, and produce picosecond wave pulses.

A photonic limiter is used to reduce the intensity of light that is emitted from the source ${ }^{123,125,126,127}$. The mechanism is to permit the passage of low intensity light, and to filter out light of higher intensity ${ }^{126,127}$. Dispersed graphene-oxide solutions are generally used for studying the optical limiter ${ }^{121,122}$. In particular, graphene, has a strong response to a change of light intensity ${ }^{125}$, with a transmittance $T$ (I) that is inversely dependent upon the light intensity I. Such a device can therefore, for example, be implemented to protect the human eye when working with laser apparatus ${ }^{123}$. Wang et al. ${ }^{126}$ also note how graphene's nonlinear response is the working principle behind the reduction of light transmitted at high intensity, and also show that graphene can limit a wide range of the visible spectrum ${ }^{126,127}$.

According to Bao et al. ${ }^{125}$, the reverse saturable absorption (opposite to saturable absorption) is the key nonlinear response that filters high intensity light, and subject to certain conditions. This relates to an optical limiter absorbing more high-energy photons than low-energy photons ${ }^{125}$. Lim et al. ${ }^{121}$ have reported that, in practice, the property will change from saturable absorption to reverse saturable absorption, only when microplasmas or microbubbles appear. These lead to a nonlinear thermal scattering, which is also an important factor in limiting high intensity light ${ }^{125}$. Nevertheless, the graphene photonic limiter is still in the early stages of development, with more drastic efforts being required in the near future.

\section{Topological Insulators}

Another even more general class of Dirac materials are Topological Insulators (TI), see for details the following Refs ${ }^{8,7,128}$. Effectively all insulators are divided in two classes: traditional insulators which do not conduct electric current and TI, which, in principle, must conduct current but it should flow only on the surface of the materials. The current may flow in the bulk but only along specific directions or specific conducting surfaces. In typical case the bulk of TI acts as an insulator, yet the surface behaves as a conductor. Electrons in the surface of a TI have zero effective mass and can travel without backscattering, even with zero resistance ${ }^{6,128}$.

Although some specific, precise examples of topological insulators were introduced in $1985^{6}$ the TI as a concept was proposed in 2005 in very insightful paper by Kane and Mele ${ }^{8}$. It was shown in Ref. 6 that on the interface between the two insulating semiconductors $\mathrm{CdTe}$ and $\mathrm{HgTe}$ (or $\mathrm{HgSe}$ ) which have correspondingly an inverted band structure with respect to CdTe a gapless Dirac spectrum may arise. The contact of two semiconductors having inverted band structure, means that the symmetry of one semiconductor conduction band corresponds to the symmetry of 


\section{Graphene Capacitor}

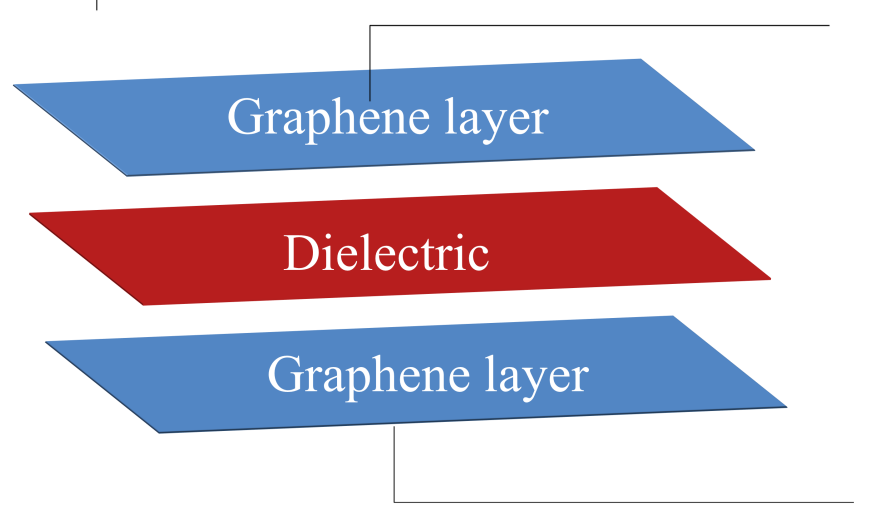

Fig. 4. Optical signaling modulator, where the graphene layers act as electrodes. This forms the basis of signaling modulator which has a fast response time and high capacitance. The size of the signaling modulator can be minimised due to graphene's atomic thickness. Furthermore, the cost of manufacturing the graphene signaling modulator is relatively inexpensive.

the valence band of the second semiconductor (or, in other words, the irreducible representation of the crystal group associated with the valence band of one semiconductor corresponds to the same irreducible representation of the conduction band of the second semiconductor) a metallic conducting layer associated with the Dirac gapless spectrum is created. The single Dirac point is protected by a time reversal symmetry and the conductivity in the Dirac point at zero temperature tends to 
infinity ${ }^{6}$. The situation may be viewed in very general terms; in the edge moving electrons are confined to a surface. When subjected to low temperatures and an external magnetic field or spin orbital interaction they experience a perpendicular Lorentz force which causes them to travel in a semi-circular trajectory along the interface at the edge. At the same time the bulk is insulating, where electrons are moving on independent circular trajectories. There all orbitals are repelling each other, very much like those found in the atomic orbitals bound to atoms in solids. The existence of the ballistic edge currents or the edge electrons moving without backscattering gives rise to the phenomenon known as the Quantum Hall Effect $(\mathrm{QHE}){ }^{129}$. For graphene there arises edge spin currents as elaborated by Kane and Mele $^{7}$.

The schematic qualitative picture of insulating states may be nicely presented with the use of the concept of circular orbitals for electron motion. So electron atomic orbitals may be viewed as the circular electron motions in the bulk of insulators. They are associated with quantised atomic energies. Due to the tunnelling between orbitals the bands and energy gaps are formed. Therewith the conduction and valence bands of traditional insulators arise. The electron orbitals may, in principle, have a size larger than the atomic ones as, for example, the cyclotron orbits in QHE. Similarly, in the bulk of IQHE, such orbits are ordered forming an insulating state where the gap is equal to the distance between Landau levels. However, if such orbitals are located at the boundary of the system they have the trajectories of the semi-circular shape. They transcend into a skipping orbit motion as the electrons rebound off the edge. The skipping orbitals create electronic states that propagate along the edge in one direction only. Because of the reflection from the boundary at certain conditions the edge states cannot therefore backscatter and as such are immune to disorder. This results in dissipationless charge flow which reminds the currents flowing in superconductors. Moreover, the edge states are chiral and have continues one sided Dirac like spectrum. The QHE occurs usually in a 2D electron gas which is created by $\delta$-doping on the GaAs/AlGaAs interface where electrons have a very high mobility and strong magnetic field applied. The IQHE was experimentally discovered in $1980^{129}$. The edge state explanation for the effect has been first developed by Buttiker in $1988^{130}$.

Considering two A and B graphene sublattices and taking two hopping integrals between next and next nearest neighbours Haldane has proposed in 1988 a model where QHE can arise even in the absence of a magnetic field. ${ }^{131}$ Later it was recognised that in semiconductor materials with strong spin-orbit (SO) coupling, there may arise a dissipationless edge spin currents, which can be induced by an electric field ${ }^{132}$. There have been predicted the existence of intrinsic spin-Hall effect ${ }^{133}$. And it was shown that in the p-doped Luttinger and Rashba models the impurity scattering vanishes. ${ }^{134,135,9}$, There exist also an interesting cancellation of the intrinsic spin Hall effect in the n-doped Rashba model. ${ }^{136}$ The experiments performed on high mobile two dimensional electron gases in GaAs are shown to be 
consistent with the above theoretical predictions. ${ }^{137}$

The concept of TI introduced by Kane and $\mathrm{Mele}^{8}$ was originally born out of the notion of the integer quantum Hall effect (IQHE) and was associated with the physics of the edge states, which always exist there, where electrons have no mass. It was recognised that there are general situations where in some insulators a certain surface (or certain 2D subsystem) may arise where an electrons mass vanishes. This creation of the Dirac spectrum and the loss of the electron mass as in the edge states of IQHE may happen even without the presence of an external magnetic field. Kane and Mele ${ }^{8}$ have predicted that due to similar reasons or due to edge states from one side as in IQHE and topological protections from other side that in graphene nanoribbons there may exist the Quantum Spin Hall Effect. The key point noticed by Kane and Mele was the spin-orbital (SO) interaction, was indeed present in graphene ${ }^{7}$. The Hamiltonian of this interaction can be presented in the form of $8 \times 8$ matrix:

$$
\hat{H}_{S O}=\Delta_{S O} \sigma_{z} \tau_{z} s_{z},
$$

where $\sigma_{z}$ is the $2 \times 2$ Pauli matrix referring to two A and B sublattices (the pseudo spin); $\tau_{z^{-}}$to two $\mathrm{K}$ and $\mathrm{K}$ ' valleys and $s_{z}$ is a Pauli matrix representing the electrons spin; $\Delta_{S O}$ is the spin-orbital (SO) coupling. Such interaction preserves both parity and time reversal symmetry and therefore must exist as an extra term in the conventional graphene Hamiltonian. The physical reason of the spin-orbit coupling in graphene are relativistic effects. Their estimation of characteristic spin-orbital coupling in graphene was about $\Delta_{S O}=2.5 \mathrm{meV}$. Obviously the heavier the atoms of which the two-dimensional lattice are made, the stronger is the spin-orbital interaction. Already for Germanene the $\mathrm{SO}$ coupling was about $\Delta_{S O}=20 \mathrm{meV}$. The importance of the spin-orbital interaction in solids was first recognised by Rashba long-ago and later he shown that the SO coupling can be important in the formation of the Dirac point of the band spectrum. Rashba has also introduced a symmetry invariant term (the Rashba term) and shown that spin-orbital interaction produces a linear energy-momentum contribution in the band spectrum, when the mirror symmetry with respect to the graphene plane is broken ${ }^{19}$. The Rashba hamiltonian has the form:

$$
\hat{H}_{\text {Rashba }}=\lambda_{\text {Rashba }}\left(\sigma_{x} \tau_{z} s_{y}-\sigma_{y} s_{x}\right)
$$

With spin orbital coupling when the Rashba term vanishes, $\lambda_{\text {Rashba }}=0$ there arises a gap in the Dirac spectrum equal to $E_{\text {gap }}=2 \Delta_{S O}$ :

$$
E= \pm \sqrt{v_{F}^{2} p_{x}^{2}+v_{F}^{2} p_{y}^{2}+\Delta_{S O}^{2}}
$$

With taking into account the Rashba term the gap decreases as $E_{g a p}=2\left(\Delta_{S O}-\right.$ $\left.\lambda_{\text {Rashba }}\right)$ and vanishes when $\Delta_{S O}=\lambda_{\text {Rashba }}$. Assuming that the Rashba term is small we note that the SO term, eq.(7), produces the gap different from all other gaps mechanisms discussed, for example, in Ref. ${ }^{30}$ or in Ref. ${ }^{7}$. The matter is that the SO gap has a different sign for each $\mathrm{K}$ and $\mathrm{K}$ ' valley. Then to connect smoothly 
between the states generated by gaps by substrate or by transverse electrical field and $\mathrm{SO}$ gap state one must go through a critical point $(\mathrm{CP})$ where there is no gap. This CP is obviously separating ground states with distinct topological orders.

At temperature well below the energy gap $E_{g}$ there may exist quantized Hall conductance, which can be calculated with the use of the conventional Kubo equation. The latter can be viewed as the topological Chern number, which may be defined with the use of the concept of Berry's curvature in reciprocal, momentum space $^{20}$.

Because the gap has an opposite sign for spin up and down electrons, an electric field applied may generate opposite currents for the opposite spins. The resulting current is the difference between the spin up and spin down currents. This gives the quantised spin Hall conductivity or the Quantum Spin Hall Effect (QSHE) ${ }^{7}$ :

$$
\sigma_{\text {spin }}=\frac{e}{2 \pi}
$$

There are also here charge currents flowing through the edge states. Note that the charge density has strong correlation with the spin density. For example, if there are charge edge currents in the graphene nanoribbon there on the both edges will arise an accumulation of the spin density. Thus, Kane and Mele shown that the spin-orbital coupling plays the role of an effective magnetic field that couples to the spin of moving electrons and that drives the ballistic charge and spin currents without backscattering, where each charge and spin conductivities are quantised ${ }^{7,8}$.

The idea of QSHE arising without Landau levels 7,8 was based on a central concept of the existence of a bulk gap and gapless edge states which form a timereversal (TR) invariant system with SO coupling. There, the left movers on the edge are correlated with down spin particles, while the right movers on the same edge have up spins. The resulting spin transport is quantized. The edge states in such QSHE have been named as Helical Liquid (HL), to reflect the fact that there are strong correlations between the spin and the momentum in the particle motion ${ }^{9}$. HL differs from chiral Luttinger Liquid (LL), because it does not break TR invariance. It differs also from spinless LL where TR transformation satisfies $T^{2}=1$ relation. It differs also from the spinfull LL. The difference between spin full LL and HL is that in the first case there are even number of TR invariants while in HL there are odd number of such invariants. Kane and Mele ${ }^{8}$ found out that HL with an even or an odd number of components are topologically distinct and are characterised by a $Z^{2}$ symmetry in the noninteracting case. Strong interactions between electrons removes strict topological distinctions between the even and odd HLs. In fact here in HL the spin orientation is determined by the direction of electron motion. HL with an odd number of components cannot be constructed in $1 \mathrm{D}^{138}$. There a uniform gap appears in the ground state when TR symmetry is spontaneously broken and a correlated two-particle backscattering term can become relevant while keeping the TR invariance. Thus, the existence of spin-orbit coupling in the system resulted in QSHE. The QSHE is associated with the time-reversalinvariant state because the relativistic effect (the origin of QSHE) does not break the 
time-reversal symmetry like an external magnetic field does. This discovery was the first step in understanding a 2D topological insulator. Like in QHE in QSHE there is an energy gap. However, at the edge states in QSHE the spin-up and spin-down electrons travel in opposite directions ${ }^{139,140,9}$.

Kane and Mele made a key theoretical advance when they proposed to use Chern number - the topological invariant or a quantity that does not change under various continuous transformations ${ }^{8}$. If such number is nontrivial (nonzero) integer, it corresponds to TI, if it is zero then it is trivial band insulator. In most cases the nontrivial integer Chern numbers are generated by spin-orbit coupling or spinorbital interaction, which was first properly understood by Rashba in $1960^{18}$. Such Chern numbers (the topological invariants) could be computed for any 2D material to predict the whether the material had a stable edge state or not. These edge states are conductors. They have a $1 \mathrm{D}$ character. There the motion of current carriers are protected from backscattering $139,140,141,142$.

Recently the QSHE or nontrivial topological insulators have been predicted to exist in silicene and two-dimensional germanium ${ }^{143}$. It may exist because the silicene nano ribbon may have topologically nontrivial electronic structures associated with nontrivial $Z^{2}$ topological invariant ${ }^{8,9,141}$. Also these materials have heavier atoms than graphene and therefore the spin-orbital coupling there is stronger. Therefore, in nanoribbons of these materials the QSHE arises even at higher temperatures than in graphene and its appearance is more pronounced.

The three-dimensional TI have been first predicated in 2007 by Fu, Kane and Mele ${ }^{11}$ and experimentally discovered by Molenkamp et $\mathrm{al}^{10}$ in the same year in $\mathrm{HgTe} / \mathrm{CdTe}$ quantum well made from two semiconductors having inverted band structure. They have used the semimetallic junctions with Dirac spectrum which was first described by Tsvelik and Kusmartsev in $1985^{6}$. Molenkamp et al have been applied transverse magnetic to make it first insulator and then studied the edge states there following to the theoretical prescription by Bernevig and Zhang ${ }^{9,141}$. Later the 3D TI have been discovered in many materials and in particular in a bismuth-antimony alloy, $\mathrm{Bi}_{x} \mathrm{Sb}_{1-x}{ }^{144}$ as it was first predicted in ${ }^{11}$. In general an arbitrary TI may have a 3D band structure which can be built by combining the band structures of ordinary insulators and Dirac spectrum having 2D. The latter arises due to topological symmetry protection, where a planar semi-metal with the Dirac spectrum is formed at the surface of the bulk insulator ${ }^{6,139,140}$.Since then many new topological insulators have been discovered. The majority of them consists of in the bismuth-selenide and bismuth-tellurium alloys. The materials exhibit TI behaviour up to higher temperatures due to the existence of the strong spinorbit coupling there. The latter arises in this materials because they have the heavy element such as Bi.

The discovery of this new class of materials; topological insulators, requires an increasing understanding of the physics associated with robust topological protection which opens a door to a new class of devices with applications includeing spintronics - electronics based on spin, and quantum computing. 
The topological insulators have the strong potential to host Majorana fermions - the real particles having fermion statistics. Because of its real character each Majorana particle is also its own antiparticle. Kitaev proposed that Majorana fermions could be used in topological quantum computations, to store bits of quantum information and would be very (topologically) stable to avoid loosing conventional decoherence $^{145}$.

\section{Weyl semimetals}

Three-dimensional materials such as $\mathrm{Ag}_{2} \mathrm{Se}$ and $\mathrm{Ag}_{2} \mathrm{Te}$ have been shown to act as small gap semiconductors ${ }^{146,147}$ and $\mathrm{Ag}_{2}$ Te can experience a phase transition from narrow-gap semiconductor to gapless semiconductor with a linear spectrum ${ }^{148}$. Other materials such as grey tin ${ }^{149}$ and mercury-telluride ${ }^{150}$ have been shown to possess zero-gap properties with a parabolic dispersion relation. In the case of mercury telluride the size of the energy gap can be adjusted by replacing atoms of mercury with the lighter element cadmium. With a specific concentration of cadmium the dispersion relation becomes gap-less and linear ${ }^{12}$.

Recently, $\mathrm{Na}_{3} \mathrm{Bi}$ has been shown to act with a three-dimensional linear dispersion relation ${ }^{13}$. The crystal structure of $\mathrm{Na}_{3} \mathrm{Bi}$ forms a hexagonal Brillouin zone in the $k_{x}-k_{y}$ plane similar to the two-dimensional material graphene. This forms threedimensional Dirac cones close to the center of the Brillouin zone.

The three-dimensional Dirac cones have also been shown in the material $\mathrm{Cd}_{3} \mathrm{As}_{2}$ $14,15,16,17$. This material possesed a non-symmetrical Dirac cone ${ }^{14}$ in the $k_{y}-k_{z}$ direction which is slightly shifted from the Fermi level ${ }^{15}$ and a Fermi-velocity 1.5 that of graphene ${ }^{16}$. These qualities make $\mathrm{Cd}_{3} \mathrm{As}_{2}$ a good candidate for the exploration of Weyl semimetals and three-dimensional Dirac cones.

It has been predicted that a new family of Weyl semimetals including TaAs, TaP, $\mathrm{NbAs}$, and NbP may posess as many as 12 pairs of Weyl points in the Brillouin zone ${ }^{151}$. Similarly to $\mathrm{Cd}_{3} \mathrm{As}_{2}$ the Weyl points appear slightly shifted from the Fermi energy; the Weyl points on the $k_{z}=0$ plane appearing at about $2 \mathrm{meV}$ above, and the off-plane Weyl points appearing about $21 \mathrm{meV}$ below the Fermi level.

In order to simply model three-dimensional materials with a linear spectrum, the two by two Weyl Hamiltonian 151,152,153:

$$
\hat{H}=v_{f} \hat{p} \cdot \vec{\sigma}+I V(x)
$$

can be used, where $v_{f}$ is the Fermi velocity, $\hat{p}$ is the three-dimensional momentum operator, $\vec{\sigma}$ is the Pauli spin matrices, $I$ is the identity matrix and $V(x)$ is an external potential. This Hamiltonian is a two by two matrix similar to that of the graphene Hamiltonian. The exception here is that for Weyl fermions momentum is not limited to the $x$ and $y$ directions. Due to the similarities with the graphene hamiltonian it is reasonable to apply the same theoretical methodologies to Weyl fermions in the hope to provide graphene like properties in a three-dimensional material. This 
Hamiltonain produces a three-dimensional graphene-like linear dispersion relation:

$$
\begin{aligned}
E=V & \pm v_{f} \sqrt{k_{x}^{2}+k_{y}^{2}+k_{z}^{2}} \\
& =V \pm v_{f} \vec{k}
\end{aligned}
$$

Here the $x$ dependent potential $V(x)$ has been replaced by a constant potential $V$. With the energy eigenvalues of the Hamiltonain, suitable eigenvectors can be selected. These eigenvectors can then be used as wave functions describing charge carriers in a Weyl semimetal and take the form:

$$
\psi=\left[\begin{array}{l}
\psi_{1} \\
\psi_{2}
\end{array}\right]=\left[\begin{array}{c}
e^{i q x+i k_{y} y+i k_{z} z} \\
\alpha e^{i q x+i \theta+i k_{y} y+i k_{z} z}
\end{array}\right]
$$

with:

$$
\begin{gathered}
q=\sqrt{\frac{(E-V)^{2}}{\hbar^{2} v_{f}^{2}}-k_{y}^{2}-k_{z}^{2}} \\
\alpha=\frac{|E-V| \sin (\phi)}{E-V+|E-V| \cos (\phi)}
\end{gathered}
$$

and the angles are defined as:

$$
\begin{gathered}
q=\frac{|E-V|}{\hbar v_{f}} \sin \phi \cos \theta \\
k_{y}=\frac{|E-V|}{\hbar v_{f}} \sin \phi \sin \theta \\
k_{z}=\frac{|E-V|}{\hbar v_{f}} \cos \phi
\end{gathered}
$$

from spherical co-ordinate theory.

\section{Scattering Properties Through a Potential Step}

The potential step described in Fig. 5 is a one-dimensional system with two distinct regions. An external potential is applied to both regions creating a scattering system with a reflection point at $x=0$.

In the potential step the initial and final mediums are not identical, therefore the transmission through the system cannot simply be taken to be $|t|^{2}$. Instead the expression for transmission must be obtained from the conservation of probability current ${ }^{154,155}$.

$$
\frac{d}{d t}|\psi|^{2}+\nabla \cdot \mathbf{j}=0
$$

As the system here is time independent only the probability current:

$$
\mathbf{j}=\psi^{*} \sigma \psi
$$




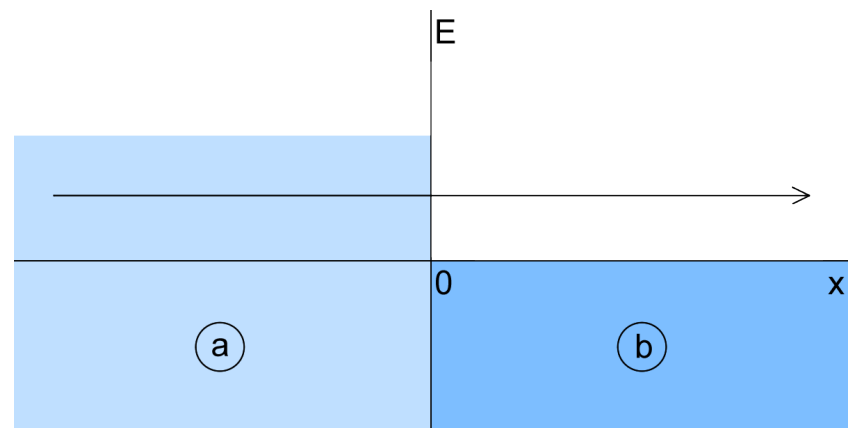

Fig. 5. Diagram of the potential step problem. A potential step is placed in the $x$-direction with a heights $V_{a} \neq 0$ and $V_{b}=0$. The shaded region shows where hole transport is present. The two independent regions have been labled as $a$ and $b$.

needs to be considered. From the continuity equation; the probability current into the system must equal the probability current out of the system. With the wave functions in Eq.(23) and Eq.(24), this results in the following expression for transmission:

$$
T=|t|^{2} \frac{\alpha_{b} \cos \left(\theta_{b}\right)}{\alpha_{a} \cos \left(\theta_{a}\right)}
$$

The subscripts $a$ and $b$ correspond to the groups of constants for the corresponding region in Fig. 5, the details of how this is obtained has been included in the supplementry information. With the expression for the transmission probability in Eq.(22) the potential step can now be solved using the wave functions in Eq.(14):

$$
\psi_{a}=\left[\begin{array}{c}
\left(e^{i q_{a} x}+r e^{-i q_{a} x}\right) e^{i k_{y} y} e^{i k_{z} z} \\
\left(\alpha_{a} e^{i q_{a} x+i \theta_{a}}-r \alpha_{a} e^{-i q_{a} x-i \theta_{a}}\right) e^{i k_{y} y} e^{i k_{z} z}
\end{array}\right]
$$

where the incident and reflected components have been included. The subscript $a$ corresponds to region $a$ in Fig. 5. The wave functions on the right of the step (corresponding to region $b$ in Fig. 5) only contain a transmitted component and is therefore given as:

$$
\psi_{b}=\left[\begin{array}{c}
t e^{i q_{b} x} e^{i k_{y} y} e^{i k_{z} z} \\
t \alpha_{b} e^{i q_{b} x+i \theta_{b}} e^{i k_{y} y} e^{i k_{z} z}
\end{array}\right]
$$

Then at the interface located at $x=0$, continuity of the wave functions require that $\psi_{a}=\psi_{b}$. Solving these simultaneous equations, with the transmission probability in Eq.(22); results in the equation:

$$
T=\frac{4 \alpha_{a} \alpha_{b} \cos \left(\theta_{a}\right) \cos \left(\theta_{b}\right)}{\alpha_{a}^{2}+\alpha_{b}^{2}+2 \alpha_{a} \alpha_{b} \cos \left(\theta_{a}+\theta_{b}\right)}
$$

The full methods used to obtain this have been included in full in the supplementry information. To obtain the plots in Fig. 6 the correct charge carrier directions must be considered. At $x=0$ there is an electron-hole interface, therefore, the right travelling charge in Fig. 5 must be carried by a left travelling hole and a right 
travelling electron. To allow for this change in direction the incident angles of the charge carriers must be changed so that $\theta_{h}=\pi-\theta_{e}{ }^{155}$ where the subscript $e$ and $h$ denote an electron or a hole respectivly. With these considerations, the result in Eq.(25) perfectly recreates the graphene result for a potential step ${ }^{154, ?}$ when the $\phi$ dependence is removed.
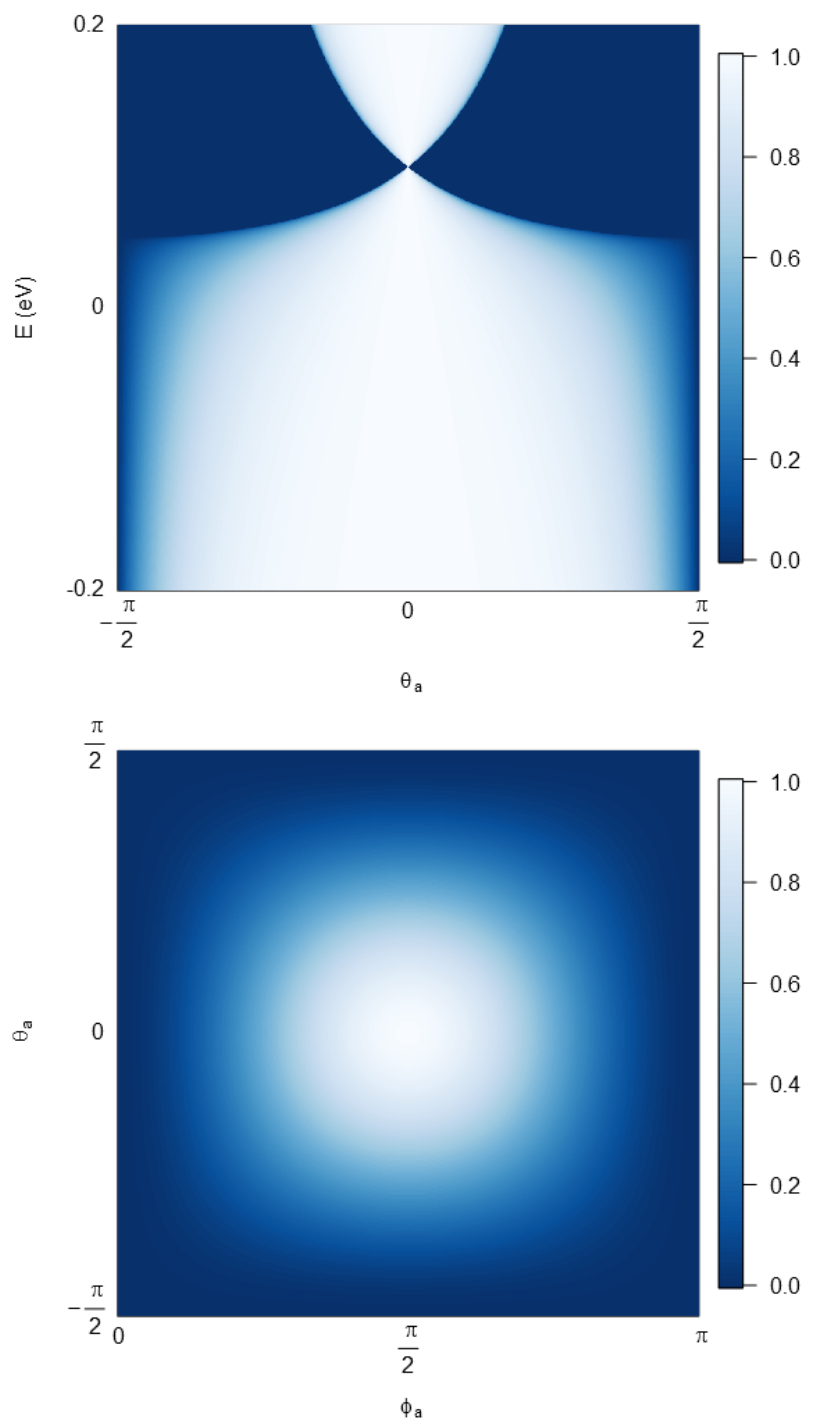

Fig. 6. Density plots for transmission against energy and incident angle for a potential step. The step shown has the heights $V_{a}=0 \mathrm{eV}$ and $V_{b}=0.1 \mathrm{eV}$. (Top) The energy dependence is then shown with $\phi_{a}=\pi / 2$. (Bottom) The angular dependence is shown in the step for an energy of $0.05 \mathrm{eV}$. 
The density plots in Fig. 6 show the symmetry between the $\theta_{a}$ and $\phi_{a}$ angles and transmission that reduces close to the step height. The top plot in Fig. 6 also shows the angular dependent transmission gap from in graphene ${ }^{157}$ appreaing in Weyl semimetals.

\section{Non-symmetrical Dirac Cones}

The materials $\mathrm{Cd}_{3} \mathrm{As}_{2}{ }^{14,15,16}$ and $\mathrm{Na}_{3} \mathrm{Bi}^{13}$ have recently been shown to possess three-dimensional Dirac cones. The experimental results show a Dirac cone with symmetry in the $k_{x}-k_{y}$ plane, however, in the $k_{x}-k_{z}$ plane there was an asymmetry. This asymmetry may cause these materials to behave differently to symmetrical Dirac cones. To model this asymmetry a scaling factor $\lambda$ can be introduced to $k_{z}$ so that $k_{z} \rightarrow \lambda k_{z}$. With this change the energy momentum relation changes to:

$$
E=V \pm v_{f} \sqrt{k_{x}^{2}+k_{y}^{2}+\lambda^{2} k_{z}^{2}}
$$

The definition of $k_{z}$ changes to:

$$
k_{z}=\frac{1}{\lambda} \frac{|E-V|}{\hbar v_{f}} \cos \phi
$$

The change in definition of $k_{z}$ in Eq.(27) will affect the scattering properties of any device constructed from these materials. The plots in Fig. 7 show the transmission probability for a potential barrier from Eq.(??) with $\lambda=1 / 3$. The scaling factor $\lambda$ causes the regions of high transmission to reduce and new regions of no transmission are introduced. The resonances that usually occur in the potential barrier become condensed into the reduced regions of transmission. In Fig. 7 the symmetry of resonances is broken, no longer showing circular resonances but a combination of resonance lines and ovals.

\section{Comparison with Optics}

The linear spectrum of Weyl fermions provides an opportunity for comparison with conventional optics. Unlike an electromagnetic wave the transmission properties of Weyl fermions cannont be entirely separated in the spacial dimensions. In the transmission probability through a barrier for Weyl fermions (Eq.(??)) there is a phase factor from the second wave function component not found in the transmission probability for an electromagnetic wave:

$$
T_{\text {optics }}=\frac{4 k^{2} q^{2}}{4 k^{2} q^{2} \cos ^{2}(q d)+\left(k^{2}+q^{2}\right)^{2} \sin ^{2}(q d)}
$$

The derivation and definitions for this can be found in the supplementary information. The similarities between $T_{\text {weyl }}$ and $T_{\text {optics }}$ are most obvious when considering that both systems experience resonance under the condition $d q=n \pi$. The resonance condition for the three-dimensional Weyl fermions expands to:

$$
E_{w e y l}=V_{b} \pm \hbar v_{f} \sqrt{\frac{n^{2} \pi^{2}}{d^{2}}+k_{y}^{2}+k_{z}^{2}}
$$




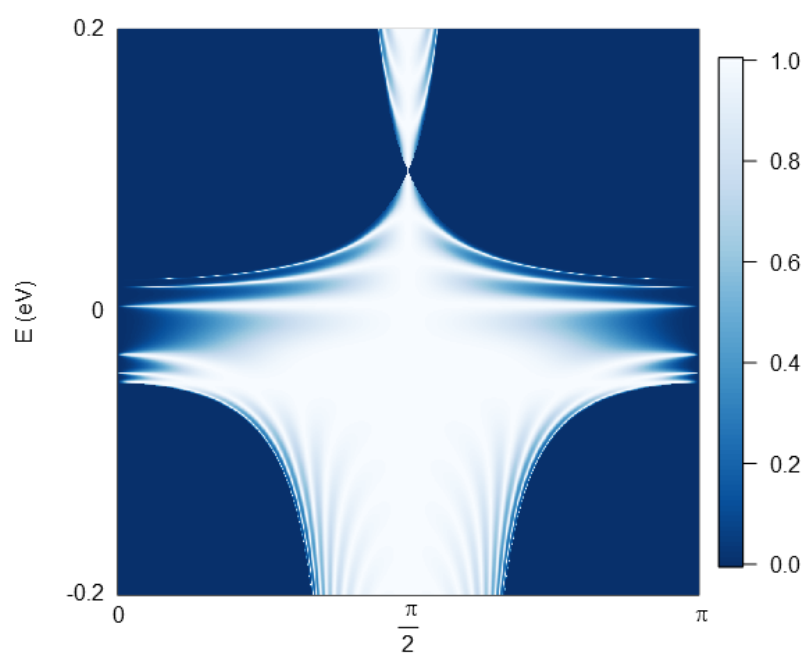

$\phi_{\mathrm{a}}$

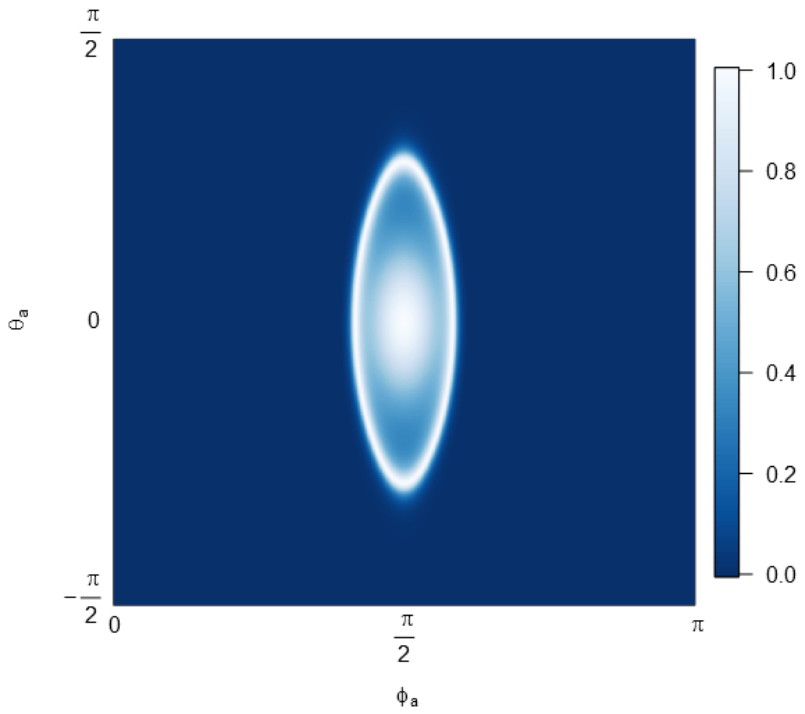

Fig. 7. Density plots for transmission probability against energy and incident angle from Eq.(??) with the modified $k_{z}$ from Eq.(27). The potential barrier shown has the characteristics $V_{a}=0 \mathrm{eV}$, $V_{b}=0.1 \mathrm{eV}$ and $\lambda=1 / 3$. (Top) The energy dependence is then shown with $\phi_{a}=\pi / 2$. (Bottom) The angular dependence is shown in the step for an energy of $0.05 \mathrm{eV}$.

and in the optical case:

$$
E_{\text {optics }}=\frac{\hbar c n \pi}{d n_{b}}
$$

Where $n_{b}$ is the refractive index of the intermediate medium. From the optical rule $q=n k$ the 'refractive index' of Weyl fermions travelling though a potential will be 
of the form:

$$
n_{w e y l}=\sqrt{\frac{(E-V)^{2}-\hbar^{2} v_{f}^{2}\left(k_{z}^{2}+k_{y}^{2}\right)}{E^{2}-\hbar^{2} v_{f}^{2}\left(k_{z}^{2}+k_{y}^{2}\right)}}
$$

However, from the probability current it is known that the transport through a potential barrier for Weyl fermions is band to band. For this reason at energies below the barrier $(E<V)$ a left travelling charge will require a left travelling electron and a right travelling hole. To represent this the refractive wave number comparison must use a different component of the wave function so that $q=-n k$, resulting in a new refractive index inside the barrier:

$$
n_{\text {weyl }}=-\sqrt{\frac{(E-V)^{2}-\hbar^{2} v_{f}^{2}\left(k_{z}^{2}+k_{y}^{2}\right)}{E^{2}-\hbar^{2} v_{f}^{2}\left(k_{z}^{2}+k_{y}^{2}\right)}}
$$

Which will be negative for all values. Using the rules for electromagnetic waves (stated in the Supplementary Information) a wavelength for the Weyl fermions may be obtained:

$$
\lambda_{w e y l}=\frac{2 \pi}{k}=\frac{2 \pi}{\sqrt{\frac{(E-V)^{2}}{\hbar^{2} v_{f}^{2}}-k_{y}^{2}-k_{z}^{2}}}
$$

With the expressions for positive/negative refractive indecies and wavelength it is possible to apply further optical analysis to the charge carriers in a Weyl semimetal. In certain conditions the Veselago lens demonstrated for charge carriers in graphene 160 may be possible in three-dimensional materials.

\section{Density of States}

The density of states can be calculated by using the general formula for density of states ${ }^{161}$ :

$$
\rho(E)=\sum_{k} \delta\left(E-E_{k}\right)
$$

where $\delta(x)$ is the Dirac delta-function. Converting sum notation to integration over all momentum:

$$
\rho(E)=\frac{L_{x} L_{y} L_{z}}{8 \pi^{3}} 2 \int_{k} \int_{\theta} \int_{\phi} \delta\left(E-E_{k}\right) k^{2} d k d \theta d \phi
$$

With $L_{x, y, z}$ being the size of the system in the respective dimension. Using the linear spectrum of Weyl fermions this can be converted to integration over energy:

$$
E_{k}=\hbar v_{f} k \quad d E_{k}=\hbar v_{f} d k \quad k^{2} d k=\frac{E_{k}^{2}}{\hbar^{3} v_{f}^{3}} d E_{k}
$$


Finally by using the integration rule $\int f(x) \delta(x) d x=f(0)$ the density of states becomes:

$$
\rho(E)=\frac{L_{x} L_{y} L_{z}}{\pi \hbar^{3} v_{f}^{3}} E^{2}
$$

Unlike in graphene; which has a linear density of states, the density of states for a three-dimensional Weyl semimetal is parabolic due to the additional $k_{z}$ component.

\section{Landauer Formalism}

The current through the scattering systems formulated earlier can be calculated with the Landauer formalism for ballistic transport. In this model perfect electron emitters are connected to a scattering device via perfectly conducting wires. The electron emitters emit electrons up to the quasi-Fermi-energy $\mu_{L}$ and $\mu_{R}$ into the respective side of the scattering device. In this model the current through the scattering device is given in Ref. 162 as:

$$
I=e v_{f} \frac{d n}{d E} T\left(\mu_{L}-\mu_{R}\right)
$$

Where $e$ is the electron charge, $v_{f}$ is the Fermi velocity and $d n / d E$ is the density of states. At a finite temperature the electron emitters inject electrons as described by the Fermi-Dirac distribution:

$$
f_{L, R}=f\left(E-\mu_{L, R}\right)=\frac{1}{e^{\frac{E-\mu_{L, R}}{k_{b} t}}+1}
$$

instead of up to the quasi-Fermi-energies $\mu_{L}$ and $\mu_{R}$. Here $k_{b}$ is the Boltzman constant and $t$ is the temperature. Using the density of states for Weyl fermions in Eq.(37) and integrating over energy and incident angle produces the $x$-direction current:

$$
I_{x}=I_{0} \int_{-\infty}^{\infty} \int_{-\pi / 2}^{\pi / 2} \int_{0}^{\pi} T(E, \theta, \phi)\left[f_{L}-f_{R}\right] E^{2} \cos (\theta) \sin (\phi) d E d \theta d \phi
$$

With the group of constants $I_{0}=e \frac{2 L_{y} L_{z}}{\pi \hbar^{3} v_{f}^{2}}$ and $L_{y, z}$ is the length of the system in the respective direction. Additional derivations can be found in the supplementary information. A suitable device has been suggested in Fig. 8; the characteristics of such a device can be seen in Fig. 9-11.

The plot of current against barrier height in Fig. 9 shows oscillations and a sharp drop in current between 0 and $0.2 \mathrm{eV}$. The drop in current is caused by the low point in transmission (shown at $E=V_{g}$ in Fig. ??) co-inciding with the energy region contained with the Fermi-Dirac distributions $f_{L}-f_{R}$. This will happen when $V_{g} \approx e V_{s d}$, outside of this region the Fermi-Dirac distributions will be centred around high transmission regions and the current will increase, as shown when $\left|V_{g}\right|>V_{s d}$.

Fig. 9 shows a similar current-voltage curve to a Zener diode with a slight bias to positive voltage. This is again due to the low point of transmission co-inciding 


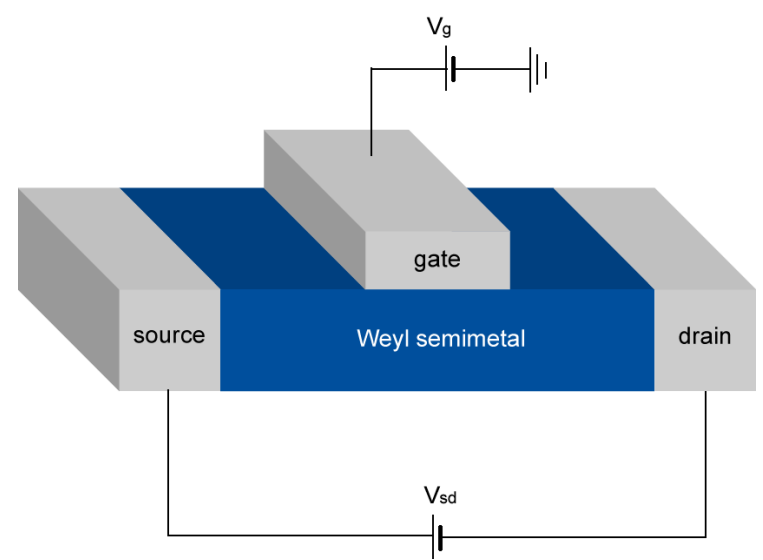

Fig. 8. A simple example of a Weyl semimetal transistor with current-voltage characteristics described by Eq.(40).

with the region contained by the Fermi-Dirac distributions. The bias can be changed to negative voltage by setting $V_{g} \rightarrow-V_{g}$, however the plateau caused by the low transmission may be moved by increasing the magnitude of the barrier.

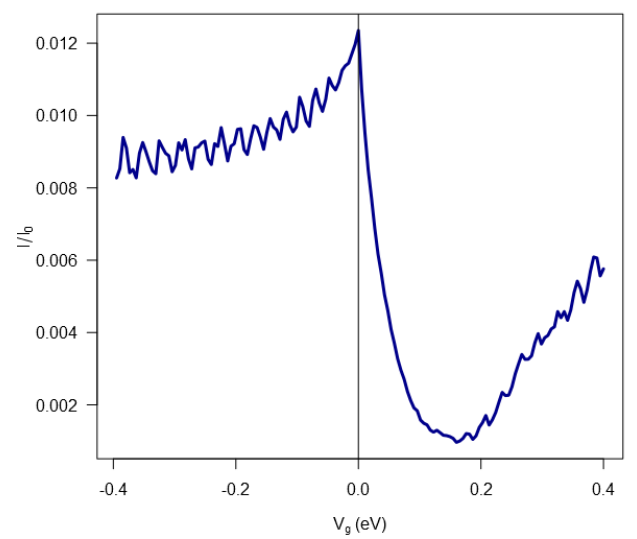

Fig. 9. The gate voltage $\left(V_{g}\right)$ dependence on current for a Weyl semimetal transistor from Eq.(40) with $I_{0}=e \frac{2 L_{y} L_{z}}{\pi \hbar^{3} v_{f}^{2}}, V_{b}=V_{g}, V_{s d}=0.2 \mathrm{eV}, d=100 \mathrm{~nm}$ and $T=298 \mathrm{~K}$.

The current steadily increases with temperature in Fig. 11, showing a parabolic dependence. As the temperature increases, less of the ballistic charge carriers will be within the energy range of the potential barrier, as fewer of the charge carriers are scattered by the barrier; the current increases. 


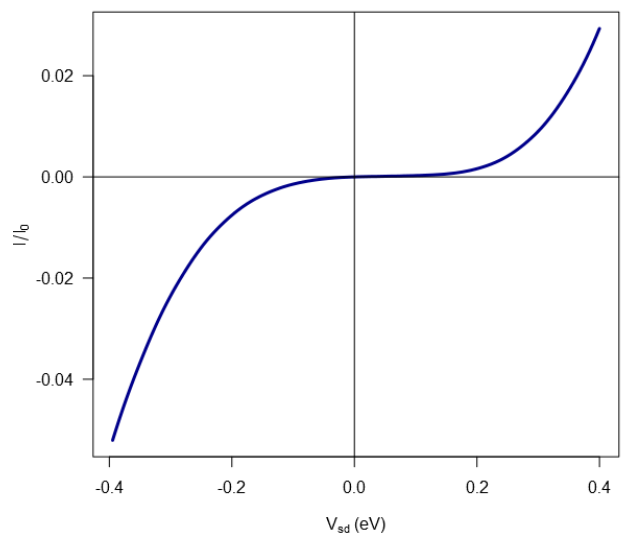

Fig. 10. The source-drain voltage $\left(V_{s d}\right)$ dependence on current for a Weyl semimetal transistor from Eq.(40) with $I_{0}=e \frac{2 L_{y} L_{z}}{\pi \hbar^{3} v_{f}^{2}}, V_{b}=0.1 \mathrm{eV}, d=100 \mathrm{~nm}$ and $T=298 \mathrm{~K}$.

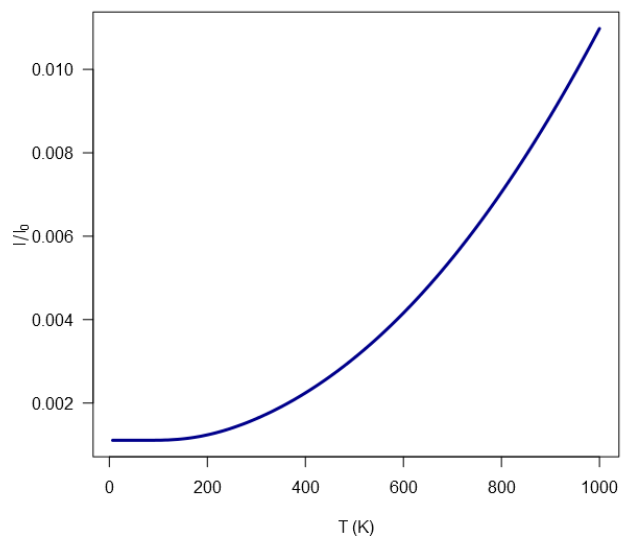

Fig. 11. The temperature dependence on current for a Weyl semimetal transistor from Eq.(40) with $I_{0}=e \frac{2 L_{y} L_{z}}{\pi \hbar^{3} v_{f}^{2}}, V_{b}=0.1 \mathrm{eV}, d=100 \mathrm{~nm}$ and $V_{s d}=0.2 \mathrm{eV}$.

\section{Conclusion}

Here we have described novel class of topological materials which include twodimensional crystals (graphene, silicene, germanene, stanene, phosphorene), two and three-dimensional topological insulators and Weyl semimetals. We have described their main outstanding properties including Klein tunnelling and Qantum Spin Hall Effect. For an illustration as an example we have described the scattering properties of a three-dimensional Dirac material, which has a linear dispersion relation such as in $\mathrm{Ag}_{2} \mathrm{Se}$ or $\mathrm{Cd}_{3} \mathrm{As}_{2}$ or TaAs and others using a three-dimensional two by two Weyl Hamiltonian.

In particular for Weyl semimetal the transmission properties through a one- 
dimensional potential step show angular symmetry between $\theta_{a}$ and $\phi_{a}$ at all energies and shares properties with the two-dimensional material graphene. The result for the potential barrier also shares many of the properties of graphene. The reduced result with $\phi_{a}=\pi / 2$ perfectly recreates the two-dimensional graphene result and features similar properties such as angle dependent transmission gap, Fabry-Pérot resonances and Klein tunnelling.

The work here aims to further the understanding of gap-less semiconductors and highlight three-dimensional materials with graphene-like properties, resulting in a guideline for the properties expected from Weyl semimetal devices.

\section{References}

1. K. S. Novoselov et al., Electric field effect in atomically thin carbon films, Science 306 (2004), pp. 666-669.

2. Geim AK, Novoselov KS. The rise of graphene. Nature Mater. 6: 183-191, 2007.

3. Meyer JC. The structure of suspended graphene sheets. Nature 446: 60-63, 2007.

4. Geim AK. Graphene: status and prospects. Science 324: 1530-1534, 2009.

5. A. H. Castro-Neto, F. Guinea, N. M. R. Peres, K. S. Novoselov and A. K. Geim, Rev. Mod. Phys., 81, 109, (2009).

6. F. V. Kusmartsev and A. M. Tsvelik, JETP lett., 42, No. 5, 257-260 (1985).

7. C. I. Kane and E.J. Mele, Phys. Rev. Lett. 95, 226801 (2005).

8. C.L. Kane and E.J. Mele, Phys. Rev. Lett. 95, 146802 (2005).

9. B. A. Bernevig, S.-C. Zhang, Phys. Rev. Lett. 96, 106802 (2006)

10. M. Konig et al, Quantum spin hall insulator state in $\mathrm{HgTe}$ quantum wells. Science 318, 766 (2007).

11. Fu, L., C.L. Kane, and E.J. Mele, Phys. Rev. Lett. 98, 106803, (2007).

12. M. Orlita, D. M. Basko, M. S. Zholudev, F. Teppe, W. Knap, V. I. Gavrilenko, N. N. Mikhailov, S. A. Dvoretskii, P. Neugebauer, C. Faugeras, A-L. Barra, G. Martinez and M. Potemski, Nature Physics, 10, (2014).

13. Z. K. Liu et al. Science, 343, 864, (2014).

14. Z. K. Liu et al., Nature Materials, 3, (2014).

15. S. Borisenko, Q. Gibson, D. Evtushinsky, V. Zabolotnyy, B. Büchner and R. J. Cava, Phys. Rev. Lett., 113, 027603, (2014).

16. M. Neupane et al. Nat. Commun. 5, No. 3786, (2014).

17. Z. Wang et al. Phys. Rev. B, 88, 125427, (2013).

18. E. I. Rashba, Fiz. Tverd. Tela (Leningrad) 2, 1224 (1960) [Sov. Phys. Solid State 2, 1109 (1960)].

19. Y.A. Bychkov and E.I. Rashba, J. Phys. C 17, 6039 (1984).

20. . D. J. Thouless, M. Kohmoto, M. P. Nightingale, and M. den Nijs, Phys. Rev. Lett. 49, 405 (1982).

21. J. M. Pereira Jr, F. M. Peeters, A. Chaves and G. A. Farias, Semicond. Sci. Technol., 25, 033002 (9pp), (2010).

22. M. I. Katsnelson, K.S. Novoselov and A. K. Geim, Nature Physics, 2, (2006).

23. Geim AK. Graphene prehistory. Phys. Scr. T146 014003: 2012, 2012.

24. Katsnelson MI. Graphene: carbon in two dimensions. Materials Today 10: 20-27, 2007.

25. Novoselov KS et al. Two-dimensional atomic crystals. PNAS 102: 10451-10453, 2005.

26. Yung K. C., Wu W. M., Pierpoint M. P., Kusmartsev F. V. Introduction 
to Graphene Electronics - A New Era of Digital Transistors and Devices. Contemp. Phys. 54, 233-251 (2013); DOI:10.1080/00107514.2013.833701,

27. Kusmartsev, F. V., et al. "Application of Graphene within Optoelectronic Devices and Transistors." Applied Spectroscopy and the Science of Nanomaterials. Springer Singapore, 191-221 (2015).

28. Savage N. Materials science: Super carbon. Nature 483: S30-S31, 2012.

29. Wallace PR. The Band Theory of Graphite. Phys. Rev. 71: 622-634, 1947.

30. O'Hare A, Kusmartsev FV, Kugel KI. A stable flat form of two-dimensional crystals: could graphene, silicene, germanene be minigap semiconductors. Nano Lett. 12: 1045-1052, (2012).

31. O'Hare, A., Kusmartsev, F. and Kugel, K., 2D Ising model with competing interactions and its application to clusters and arrays of TT-Rings, graphene and adiabatic quantum computing, International Journal of Modern Physics B23, 3951 (2009).

32. A. O'Hare, F. V. Kusmartsev and K. I. Kugel, Stable forms of two-dimensional crystals and graphene, PHYSICA B-Cond. Matt., 407 (2012) pp. 1964-1968, DOI: 10.1016/j.physb.2012.01.075.

33. G. G. Guzman-Verri and Lew Yan Voon, Phys. Rev. B 76, 075131 (2007).

34. P. Vogt, et al. Phys. Rev. Lett. 108, 155501 (2012).

35. Davila, M. E., Xian, L., Cahangirov, S., Rubio, A. and Le Lay, G., New J. Phys. 16, 095002 (2014).

36. Kim, J.-S. et al. Preprint at http://arxiv.org/abs/1412.0355 (2014).

37. S. Chen, Thermal conductivity of isotopically modified graphene, Nature Mater. 11 (2012), pp. 203-207.

38. J. Rafiee, Wetting transparency of graphene, Nature Mater. 11(2012), pp. 217-222 .

39. K. S. Novoselov et al., Room-temperature quantum hall effect in graphene, Science 315 (2007), p. 1379

40. M. I. Katsnelson, Graphene: carbon in two dimensions, Materials Today 10 (2007), pp. $20-27$.

41. A. Pototsky, F. Marchesoni, F.V. Kusmartsev, P. Hanggi and S. E. Savel'ev, Relativistic Brownian motion on a graphene chip, European Physical Journal B 85 (2012), p. 356.

42. V. V. Zalipaev, D. N. Maksimov, C. M. Linton and F.V. Kusmartsev, Spectrum of localized states in graphene quantum dots and wires, Phys. Lett. A 377 (2013), pp. 216-221.

43. Y. Iyechika, Application of graphene to high-speed transistors: expectations and challenges, Science and Technology Trends - Quarterly Review 37 (2010), pp. 76-92.

44. Tao, L. et al. Nature Nanotechnol. http://dx.doi.org/10.1038/NNANO.2014.325 (2015).

45. J. Bardeen and W. H. Brattain, The transistor, a semiconductor triode, Phys. Rev. 74 (1948), pp. 230-231.

46. F. Schwierz, Graphene transistors, Nature Technology 5 (2010), pp. 487-496.

47. N. Savage, Super Carbon, Nature 483 (2012), S30-S31.

48. P. R. Wallace, Band theory of graphite, Phys. Rev. 71 (1947), pp. 622-634.

49. D. R. Dreyer, R. S. Ruoff and C. W. Bielawski, From conception to realization: an historical account of graphene and some perspectives for its future, Angew. Chem. Int. Ed. 49 (2010), pp. 9336-9344.

50. V. G. Ruess and F. H. Vogt, Hchstlamellarer Kohlenstoff aus Graphitoxyhydroxyd, Monatshefte fr Chemie 78 (1948), pp. 222-242.

51. H. P. Boehm, A. Clauss, G. O. Fischer and U. Hofmann, Das Adsorptionsverhalten Sehr Dunner Kohlenstoff-Folien, Z. Anorg. Allg. Chem. 316 (1962), pp. 119-127. 
52. H. P. Boehm, A. Clauss, U. Hofmann and G. O. Fischer, Dunnste Kohlenstoff-Folien, Zeitschrift Fur Naturforschung Part B-Chemie Biochemie Biophysik Biologie Und Verwandten Gebiete. B 17 (1962), pp. 150-153.

53. H. R. Gall, E. V. Rutkov and A. Y. Tontegode, Two dimensional graphite films on metals and their intercalation, Int. J. Mod. Phys. B 11 (1997), pp. 1865-1911.

54. C. Berger et al., Ultrathin epitaxial graphite: 2d electron gas properties and a route toward graphene-based nano-electronics, J. Phys. Chem. B 108 (2004), pp. 1991219916.

55. M. L. Sadowski, G. Martinez, M. Potemski, C. Berger and W. A. de Heer, Magnetospectroscopy of epitaxial few-layer graphene Solid State Comm. 143 (2007), pp. 123125.

56. S. Novoselov et al., Two-dimensional atomic crystals, PNAS 102 (2005), pp. 1045110453.

57. W. A. de Heer et al., Epitaxial graphene, Solid State Comm. 143 (2007), pp. 92-100.

58. K. V. Emtsev et al., Towards wafer-size graphene layers by atmospheric pressure graphitization of silicon carbide, Nature Mater. 8 (2009), pp. 203-207.

59. Z. P. Chen, W. Ren, L. Gao, B. Liu, S. Pei and H. Cheng, Three-dimensional flexible and conductive interconnected graphene networks grown by chemical vapor deposition, Nature Mater. 10 (2011), pp. 424-428.

60. K. S. Kim et al., Large-scale pattern growth of graphene films for stretchable transparent electrodes, Nature 457 (2009), pp. 706-710.

61. X. Li et al., Large-area synthesis of high-quality and uniform graphene films on copper foils, Science 324 (2009), pp. 1312-1314.

62. G. Hlawacek et al., Imaging ultra-thin layers with helium ion microscopy: utilizing the channeling contrast mechanism, Beilstein J. Nanotechnol. 3 (2012), pp. 507-512.

63. J. A. Robinson et al. Correlating raman spectral signatures with carrier mobility in epitaxial graphene: a guide to achieving high mobility on the wafer scale, Nano Lett. 9 (2009), pp. 2873-2876.

64. A. Ben Gouider Trabelsi, A. Ouerghi, O.E. Kusmartseva, F.V. Kusmartsev, M. Oueslati, Raman Spectroscopy of four epitaxial graphene layers: Macro-island grown on 4H-SiC (000) substrate and an associated strain distribution, Thin Solid Films 539, 377-383, (2013).

65. Trabelsi, A. B. G., Kusmartsev, F. V., Robinson, B. J., Ouerghi, A., Kusmartseva, O. E., Kolosov, O. V., ... Oueslati, M. Charged nano-domes and bubbles in epitaxial graphene. Nanotechnology, 25, 165704, (2014)

66. R. Bkakri, O.E. Kusmartseva, F.V. Kusmartsev, M. Song, A. Bouazizi, Degree of phase separation effects on the charge transfer properties of P3HT: Graphene nanocomposites, Journal of Luminescence, 161, 264 ?270 (2015)

67. R. Bkakri, O.E. Kusmartseva, F.V. Kusmartsev, M. Song, L. Sfaxi, A. Bouazizi, Charge transfer properties in P3HT: graphene capped InAs/GaAs QDs hybrid heterostructure for photovoltaic application, Synthetic Metals, 203, 74 ?81 (2015)

68. J. H. Chen, C. Jang, S. Xiao, M. Ishigami and M. S. Fuhrer, Intrinsic and extrinsic performance limits of graphene devices on $\mathrm{SiO}_{2}$, Nature Nanotechnol 3 (2008), pp. 206-209.

69. K. I. Bolotin et al., Ultrahigh electron mobility in suspended graphene, Solid State Commun. 146 (2008), pp. 351-355.

70. S. Bae et al., Roll-to-roll production of 30-inch graphene films for transparent electrodes, Nature Nanotechnol. 5 (2010), pp. 574-578.

71. X. Du, I. Skachko, A. Barker and E. Y. Andrei, Approaching ballistic transport in suspended graphene, Nature Nanotechnol. 3 (2008), pp. 491-495. 
72. S. V. Morozov et al. Giant intrinsic carrier mobilities in graphene and its bilayer, Phys. Rev. Lett. 100 (2008), p. 016602.

73. F. V. Kusmartsev and A. M. Tsvelik, Semi-metallic properties of a heterojunction, JETP Lett. 42 (1985), pp. 257-260.

74. H. S. Song et al., Origin of the relatively low transport mobility of graphene grown through chemical vapor deposition, Sci. Rep. (Nature) 2 (2012), p. 337.

75. S. D. Sarma, S. Adam, E. H. Hwang and E. Rossi, Electronic transport in twodimensional graphene, Rev. Mod. Phys. 83 (2011), pp. 407-470.

76. S. Datta, 1Electronic Transport in Mesoscopic Systems. Cambridge: Cambridge University Press, 1997.

77. Yang-Bo Zhou, Han-Chun Wu, Da-Peng Yu, and Zhi-Min Liao, Magnetoresistance in graphene under quantum limit regime, Appl. Phys. Lett. 102, (2013) 093116.

78. M. E. Pumarol et al., Direct nanoscale imaging of ballistic and diffusive thermal transport in graphene nano-structures, Nano Lett. 12 (2012), pp. 2906-2911.

79. R. Prasher, Graphene spreads the heat, Science 328 (2010), pp. 185-186.

80. A. A. Balandin et al., Superior Thermal conductivity of Single-Layer Graphene, Phys. Rev. Lett. 8 (2008), pp. 902-907.

81. J. H. Seol et al., Two-Dimensional Phonon Transport in Supported Graphene, Science 328 (2010), pp. 213-216.

82. Y. K. Koh, M. H. Bae, D. G. Cahill and E. Pop, Heat conduction across monolayer and few-layer graphenes, Nano Lett. 10 (2010), pp. 4363-4368.

83. R. R. Nair et al., Fine structure constant defines transparency of graphene, Science 320 (2008), pp. 1308-1308.

84. F. Bonaccorso, Z. Sun, T. Hasan and A. C. Ferrari, Graphene photonics and optoelectronics, Nature Photonics 4 (2010), pp. 611-622.

85. R. J. Stoehr, R. Kolesov, J. Pflaum, J. and J. Wrachtrup, Fluorescence of laser created electron-hole plasma in graphene, Phys. Rev. B 82 (2010), p. 121408.

86. M. Breusing, C. Ropers and T. Elsaesser, Ultrafast carrier dynamics in graphite, Phys. Rev. Lett. 102 (2009), p. 086809.

87. T. Kampfrath, L. Perfetti, F. Schapper, C. Frischkorn and M. Wolf, Strongly coupled optical phonons in the ultrafast dynamics of the electronic energy and current relaxation in graphite, Phys. Rev. Lett. 95 (2005), p. 187403.

88. M. Lazzeri, S. Piscanec, F. Mauri, A. C. Ferrari and J. Robertson, Electronic transport and hot phonons in carbon nanotubes, Phys. Rev. Lett. 95 (2005), p. 236802.

89. Z. Jiang, Y. Zhang, Y. W. Tana, H. L. Stormer and P. Kim, Quantum Hall effect in graphene, Solid State Comm. 143 (2007), pp. 1419

90. M. I. Katsnelson, K. S. Novoselov and A. K. Geim, Chiral tunneling and the klein paradox in graphene, Nature Phys. 2 (2006), pp. 620-625.

91. A. Calogeracos, Paradox in a pencil, Nature Phys. 2 (2006), pp. 579-580.

92. R. K. Su, G. G. Siu and X. Chou, Barrier penetration and klein paradox, J. Phys A: Math. Gen. 26 (1993), pp. 1001-1005.

93. A. Calogeracos and N. Dombey, History and physics of klein paradox, Contemp. Phys. 40 (1999), pp. 313-321.

94. R. R. Hartmann, N. J. Robinson, and M. E. Portnoi, Smooth electron waveguides in graphene. Phys. Rev. B 81 (2010) pp. 245431.

95. Z. Wu, Appl. Phys. Lett. 98 (2011) pp. 082117.

96. J. R. Williams, T. Low, M. S. Lundstrom, and C. M. Marcus, Nat. Nanotech. 6 (2011) pp. 222.

97. C. A. Downing, D. A. Stone, and M. E. Portnoi, Zero-energy states in graphene quantum dots and rings, Phys. Rev. B 84 (2011) pp. 155437. 
98. Stone, D. A.; Downing, C. A.; Portnoi, M. E., Searching for confined modes in graphene channels: The variable phase method, Phys. Rev. B 86, (2012) pp. 075464.

99. F.V. Kusmartsev, Fine-Structure and Fractional $M / N$ Aharonov-Bohm Effect, Phys. Rev. B52 (1995) pp. 14445-14456.

100. Liu, Yang; Brada, M.; Mele, E. J.; et al., Annalen der Physik, 526, 449 (2014).

101. R. D. Y. Hills and Kusmartsev, F. V., Annalen der Physik, 526, 437 (2014).

102. D.M. Forrester and Kusmartsev, F. V., Nanoscale, 6, 7594 (2014).

103. G. L. Yu et al. Interaction phenomena in graphene seen through quantum capacitance, PNAS 110 (2013), pp. 3282-3286.

104. J. Xia, F. Chen, J. Li and N. Tao, Measurement of the quantum capacitance of graphene, Nature Nanotech. 4 (2009), pp. 505-509

105. F. Giannazzo, S. Sonde, V. Raineri and E. Rimini Screening length and quantum capacitance ing graphene by scanning probe microscopy, Nano Lett. 9, pp. 23-29 (2009).

106. P. Avouris, Z. Chen and V. Perebeinos, Carbon-based electronics, Nature Nanotech. 2 (2007), pp. 605-615.

107. X. Li, X. Wang, L. Zhang, S. Lee and H. Dai, Chemically derived, ultra-smooth graphene nanoribbon semiconductors, Science 319 (2008), pp. 1229-1232.

108. J. Park, S. Nam, M. Lee and C. M. Lieber, Synthesis of monolithic graphene-graphite integrated electronics, Nature materials 98 (2011), p. 082117.

109. Q. He, S. Wu, Z. Yin and H. Zhang, Graphene-based electronic sensors, Chem. Sci. 3 (2012), pp. 1764-1772.

110. Q. He et al., Transparent, flexible, all-reduced graphene oxide thin film transistors, ACS Nano 5 (2011), p. 082117.

111. H. W. Zhu, C. L. Xu, D. H. Wu, B. Q. Wei, R. Vajtai, P. M. Ajayan, Direct Synthesis of Long Single-Walled Carbon Nanotube Strands, Science 296 (2002), pp. 884-886.

112. T. H. Hewett, F. V. Kusmartsev, Extraordinary magnetoresistance: sensing the future, Central European Journal of Physics, 10 (2012), pp 602-608.

113. M. Engel et al., Light matter interaction in a micro-cavity controlled graphene transistor room temperature transistor based on a single carbon nanotube, Nature Commun. 3 (2012), pp. 906-911.

114. Gosciniak J, Tan DTH. Theoretical investigation of graphene-based photonic modulators. Scientific Reports 3: 1897, 2013.

115. Liu M et al. A graphene-based broadband optical modulator. Nature 474: 64-67, 2011.

116. Zhang FM, He Y, Chen X. Guided modes in graphene waveguides. Appl. Phys. Lett., 94: 212105, 2009.

117. Wu Q, Turpin JP, Werner DH. Integrated photonic systems based on transformation optics enabled gradient index devices. Light: Science and Applications 38, 2012 .

118. Kim JT, Choi SY. Graphene-based plasmonic waveguides for photonic integrated circuits. Optic Express 19: 24557-24562, 2011.

119. Wang X, Cheng Z, Xu K, Tsang HK, Xu JB. High-responsivity graphene/silicon-heterostructure waveguide photodetectors. Nature Photonics 7: 888891, 2013.

120. Avouris P, Xia FN. Graphene applications in electronics and photonics. Material Research Soc. 37: 1225-1234, 2012.

121. Lim GK et al. Giant broadband nonlinear optical absorption response in dispersed graphene single sheets. Nature Photonics 5: 554-560, 2011.

122. Bao Q et al. Monolayer graphene as a saturable absorber in a mode-locked laser. Nano Res. 4(3):297-307, 2011. 
123. Bonaccorso F, Sun Z, Hasan T, Ferrari AC. Graphene photonics and optoelectronics. Nature Photonics 4: 611-622, 2010.

124. Hendry E, Hale P, Moger J, Savchenko A, Mikhailov S. Coherent nonlinear optical response of graphene. Phys. Rev. Lett. 105: 97401, 2010.

125. Bao Q, Loh KP. Graphene photonics, plasmonics, and broadband optoelectronic devices. ACS Nano, 6: 3677-3694, 2012.

126. Wang J, Hernandez Y, Lotya M, Coleman JN, Blau WJ. Broadband Nonlinear Optical Response of Graphene Dispersions. Adv. Mater. 21: 2430-2435, 2009.

127. Tutt LW, Kost A. Optical limiting performance of C60 and C70 solutions. Nature 356: 225-226, 1992.

128. J. E. Moore, Nature, 164, pp. 194-198, (2010).

129. K. Von Klitzing, G. Dorda and M. Pepper, Phys. Rev. Lett. 45, 494 (1980).

130. M. Buttiker, Phys. Rev. B 38, 9375 (1988)

131. F.D.M. Haldane, Phys. Rev. Lett. 61, 2015 (1988).

132. S. Murakami, N. Nagaosa, and S. Zhang, Science 301, 1348 (2003).

133. J. Sinova et al., Phys. Rev. Lett. 92, 126603 (2004).

134. S. Murakami, Phys. Rev. B 69, 241202(R) (2004).

135. B.A. Bernevig et al., Phys. Rev. Lett. 95, 016801 (2005).

136. J. Inoue et al., Phys. Rev. B 70, 041303 (2004).

137. Y. Kato et al., Phys. Rev. Lett. 93, 176601 (2004).

138. C. Wu, B. A. Bernevig, and S.-C. Zhang, Phys. Rev. Lett. 96, 106401 (2006).

139. D. Kong Y. Cui, Opportunities in Chemistry and Materials Science for Topological Insulators and their Nanostructures, Nature Chemistry, Vol. 3, pp. 845-849 (2011);

140. J. E. Moore C. Kane, Topological Insulators, Physics World, 24, 32-36 (2011).

141. B. A. Bernevig,T. L. Hughes,S.-C. Zhang, Quantum spin Hall effect and topological phase transition in HgTe quantum wells. Science 314, 1757 (2006).

142. C. Liu et al, Quantum spin Hall effect in inverted type-II semiconductors. Phys. Rev. Lett. 100, 236601 (2008).

143. Liu, Cheng-Cheng, W. Feng, and Yugui Yao. "Quantum spin Hall effect in silicene and two-dimensional germanium." Phys. Rev. Lett. 107, 076802, (2011).

144. Pedram Roushan, Jungpil Seo, Colin V. Parker, Y. S. Hor, D. Hsieh, Dong Qian, Anthony Richardella, M. Z. Hasan, R. J. Cava and Ali Yazdani,Topological Surface States Protected From Backscattering by Chiral Spin Texture, Nature 460, 1106-1109 (2009).

145. A.Y. Kitaev, Phys. Usp. 44, 131, (2001).

146. R. Xu, A. Husmann, T. F. Rosenbaum, M.-L Saboungi, J. E. Enderby and P. B. Littlewood, Nature, 390, (1997).

147. R. Dalven and R. Gill, Physical Review, 159, No. 3, (1967).

148. A. A. Abrikosov, J. Phys. A: Math. Gen., 36, 9119-9131, (2003).

149. S. Groves and W. Paul, Phys. Rev. Lett., Vol. 11, No. 5, (1963).

150. N. N. Berchenko and M. V. Pashkovskii, Sov. Phys. Usp., Vol. 19, No. 6, (1976).

151. H. Weng, C. Fang, Z. Fang, B. A. Bernevig and X. Dai, Phys. Rev. X, 5, 011029, (2015).

152. X. Wan, A. M. Turner, A. Vishwanath and S. Y. Savrasov, Phys. Rev. B, 83, 205101, (2011).

153. S. M. Young, S. Zaheer, J. C. Y. Teo, C. L. Kane, E. J. Mele and A. M. Rappe, Phys. Rev. Lett., 108, 140405, (2012).

154. M.R. Setare, D. Jahani, J. Phys.: Condens. Matter, 22, 245503, (2010).

155. T. Tudorovskiy, K. J. A. Reijnders and M. I. Katsnelson Phys. Scr., T146, 014010, (17pp), (2012). 
156. R. D. Y. Hills and F. V. Kusmartsev, Ann. Phys. (Berlin), 526, No. 910, 437448, (2014).

157. X. Chen and J. Tao, Appl. Phys. Lett. 94, 262102, (2009).

158. Wave Propagation: From Electrons to Photonic Crystals and Left-Handed Materials, P. Markos and C. M. Soukoulis, Princeton University Press, 1 Apr 2008.

159. M. R. Masir, P. Vasilopoulos and F. M. Peeters, Phys. Rev. B, 82, 115417, (2010).

160. V. V. Cheianov, V. Falko and B. L. Altshuler Science, 315, p.1252, (2007).

161. V. A. Yampol'skii, S. Savel'ev and F. Nori, New J. Phys., 10, 053024, (2008).

162. M. Buttiker, Y. Imry, R. Landauer and S. Pinhas, Phys. Rev. B, 31, No. 10, (1985). 